\title{
Geographical distribution, abundance and some population characteristics of the species of the genus Pagellus (Osteichthyes: Perciformes) in different areas of the Mediterranean*
}

\author{
MARIA TERESA SPEDICATO ${ }^{1}$, SILVESTRO GRECO², KOSTAS SOPHRONIDIS ${ }^{3}$, \\ GIUSEPPE LEMBO ${ }^{1}$, DANIELA GIORDANO ${ }^{2}$ and ANNA ARGYRI ${ }^{3}$ \\ ${ }^{1}$ COISPA Tecnologia \& Ricerca, Via dei Trulli 18-20, 70045 Bari-Torre a Mare, Italy. E-mail: coispa@eostel.it \\ ${ }^{2}$ Istituto Sperimentale Talassografico, C.N.R., Spianata S. Raineri 86, 98122 Messina, Italy. \\ ${ }^{3}$ Fisheries Research Institute-NAGREF, N. Peramos 64007, Kavala, Greece.
}

\begin{abstract}
SUMMARY: The objective of this paper was to investigate the local distribution, abundance and population structure of Pagellus erythrinus, Pagellus bogaraveo and Pagellus acarne on the continental shelf and slope over a wide geographic area from Gibraltar to the Aegean Sea. The analysis was based on the data collected during six trawl surveys, from 1994 to 1999, carried out yearly in spring within the MEDITS EU project. P. acarne was mainly distributed on the continental shelf, with a relatively higher abundance in the western part of the Mediterranean basin, where the biomass indices of $P$. bogaraveo were also important on the continental slope. Throughout the studied area, P. erythrinus was mainly distributed on the continental shelf, with remarkable abundance indices in the eastern part of the Mediterranean and in Corsica's waters. Mean total lengths showed a highly significant decreasing trend from the western to the eastern geographical sectors.
\end{abstract}

Key words: Pagellus acarne, Pagellus erythrinus, Pagellus bogaraveo, Mediterranean Sea, abundance indices, mean length.

\section{INTRODUCTION}

Pagellus acarne (Risso, 1826), Pagellus bogaraveo (Brünnich, 1768) and Pagellus erythrinus (Linnaeus, 1758) are three widely distributed Sparidae species whose distribution area encompasses both hemispheres, from Scandinavia to Senegal and Angola (Tortonese, 1975), and represent the target of different fisheries (trawl, longline, gillnet). In the East Atlantic these species are of great commercial interest and are common in the small-scale fisheries. Thus, aspects of their population biology and fishery have been investigated in different areas (e.g. Santos

\footnotetext{
*Received November 13, 2000. Accepted January 27, 2002.
}

et al., 1995; Erzini et al., 1998; Pajuelo and Lorenzo, 1998; Sousa et al., 1999).

Also in the Mediterranean, these fishes represent appreciated fishery resources (Abellan and Basurco, 1999) and occur throughout the basin (P. erythrinus also in the Black Sea), although with difference in frequency of occurrence and relative abundance between the western and eastern regions. The Axillary Seabream (P. acarne) and the Common Pandora (P. erythrinus) preferably inhabit the continental shelf bottoms, while the Blackspot Seabream ( $P$. bogaraveo) is commoner on the slope. In some areas, a size-dependent distribution by depth has also been reported for the three species, with the juveniles appearing mainly concentrated in shallow- 
er waters (e.g. Orsi Relini et al., 1984; Orsi Relini and Fida, 1992).

These species are generally recognised as being hermaphroditic, proterandric ( $P$. acarne and $P$. bogaraveo) and proterogynous (P. erythrinus). Nevertheless, their sexual pattern seems to be characterised by a considerable complexity, and gonocoric individuals, as well as primary females or males, are frequently identified (D'Ancona, 1949; Larrañeta, 1964; Lissia Frau, 1968; Sánchez, 1983; Krug, 1990). The amount of information available on their biology and population dynamics varies considerably among the three species and geographical regions. Biological characteristics and demographic parameters of $P$. erythrinus, such as maturity, reproductive season, recruitment time, growth and mortality, have been investigated in different areas of the western Mediterranean (e.g. Larrañeta, 1964, 1967; Girardin and Quignard, 1985; Orsi Relini and Romeo, 1985; Abella et al., 1997), central Mediterranean (Rijavec and Zupanovic, 1965; Jukic and Arneri, 1984; Andaloro and Prestipino Giarritta, 1985; Jarboui et al., 1998) and eastern Mediterranean (Livadas, 1988; Mytilineou, 1988; Vassilopoulou et al., 1986). There is also some information on P. acarne and P. bogaraveo, but it is more limited in terms of areas and topics, such as reproductive biology and growth (Andaloro, 1983a, b; Campillo, 1992; Orsi Relini and Fida, 1992; Stergiou et al., 1997). Moreover, the interest of the different Pagellus species for aquaculture also stimulated studies on their reproduction, feeding and growth (Cejas et al., 1993; Greco et al., 1995; Micale and Genovese, 1998; Spedicato et al., 1998).

Depending on the species, more or less detailed information related to the biology and population dynamics is thus available for some geographical zones. Nevertheless, data on large-scale geographical distribution and relative abundance, as well as on population characteristics, are scarce. The objective of this paper was to carry out a preliminary analysis and comparison of different geographical areas from Gibraltar to the Aegean Sea by investigating the local distribution, abundance and population size structure of $P$. erythrinus, $P$. bogaraveo and $P$. acarne, on the continental shelf and upper and middle slope. Data collected by trawl surveys throughout the Mediterranean using the same methodology and gear (Bertrand et al., 2000, 2002) represent a particularly suitable base for comparing abundance and length structure among several geographical regions and over time (Pennington and Strømme, 1998).

\section{MATERIAL AND METHODS}

Among the 30 reference species of the European Union research project "MEDITS" (Bertrand et al., 2000, 2002), six members of the Sparidae family were included. Of these, only the three species examined in this study were considered as target species from the beginning of the project (1994), and they were therefore selected for the present study since the time series on their distribution and population characteristics was more complete. The analysed data were collected from 1994 to 1999, during six trawl surveys carried out yearly in spring (May-June) within the framework of the above mentioned project. The sampling procedures were standardised and a common protocol was applied. Further details on the stratification scheme (stratified random sampling with five bathymetric limits: 50, 100, 200, 500 and $800 \mathrm{~m}$; each haul position randomly selected in small sub-areas and maintained fixed throughout the time), area subdivision in geographical sectors, and sample allocation (proportional to each depth stratum area) by geographical sector and year (1994-1999) are given in Bertrand et al. (2000, 2002).

The same gear (GOC 73, by P.Y. Dremière, IFREMER-Sète), with a $20 \mathrm{~mm}$ stretched mesh size in the cod-end was employed in the different geographical zones. Data on the gear characteristics, operational parameters and performance are reported in Dremière and Fiorentini (1996). The horizontal net opening ranged between 14 and $19.5 \mathrm{~m}$, according to the warp length, which in turn depended on the sampling depth. When the wing spread could not be directly measured it was estimated by the asymptotic relationship proposed by Souplet (1996), on the basis of a wide set of experimental data. The hauls were carried out during daylight only and each lasted 30 minutes in the $10-200 \mathrm{~m}$ strata and 60 minutes in the strata deeper than $200 \mathrm{~m}$.

Total catches of the three Pagellus species were weighed and all the individuals counted. Thus, the abundance indices (biomass and density) were calculated in the standardised form $\left(\mathrm{kg} \cdot \mathrm{km}^{-2}\right.$; Number $\cdot \mathrm{km}^{-2}$, respectively) on the basis of the following parameters: $\mathrm{kg}$ or number of individuals, distance covered and wing spread of each tow ("swept area method"; Pauly, 1983).

The length data (total length) were collected on board, with the individuals measured to the lower half centimetre. Length frequency distributions were constructed for each geographical sector and depth 
TABLE 1. - Occurrence of Pagellus acarne, Pagellus bogaraveo and Pagellus erythrinus in the MEDITS hauls (all surveys 1994-1999). Total occurrence and percentage for shelf and slope.

\begin{tabular}{|c|c|c|c|c|c|c|c|c|c|c|}
\hline \multirow{3}{*}{ Geographical sector } & \multicolumn{3}{|c|}{ Pagellus acarne } & \multicolumn{3}{|c|}{ Pagellus bogaraveo } & \multicolumn{3}{|c|}{ Pagellus erythrinus } & \multirow{3}{*}{$\begin{array}{l}\text { Number } \\
\text { of hauls }\end{array}$} \\
\hline & Total & shelf & slope & Total & shelf & slope & Total & shelf & slope & \\
\hline & rence & $\%$ & $\%$ & occurrence & $\%$ & & occurrence & $\%$ & $\%$ & \\
\hline SE Adriatic-Albania & 9 & 88.89 & 11.11 & 10 & 60.00 & 40.00 & 23 & 95.65 & 4.35 & 160 \\
\hline Alboran Sea-Alicante-Catalan Sea & 204 & 79.90 & 20.10 & 153 & 41.83 & 58.17 & 134 & 100.00 & 0.00 & 614 \\
\hline Gulf of Lions-Corsica & 170 & 86.47 & 13.53 & 90 & 53.33 & 46.67 & 163 & 93.87 & 6.13 & 548 \\
\hline N Aegean Sea & 28 & 60.71 & 39.29 & 52 & $21: 15$ & 78.85 & 32 & 96.88 & 3.13 & 344 \\
\hline Argosaronikos & 40 & 90.00 & 10.00 & 38 & 52.63 & 47.37 & 79 & 98.73 & 1.27 & 241 \\
\hline S Aegean Sea & 64 & 76.56 & 23.44 & 73 & 13.70 & 86.30 & 74 & 97.30 & 2.70 & 323 \\
\hline NE Adriatic-Croatia & 19 & 94.74 & 5.26 & 16 & 93.75 & $6: 25$ & 81 & 100.00 & 0.00 & 151 \\
\hline Ligurian, $\mathrm{N}$ and Central Tyrrhenian Sea & 140 & 85.00 & 15.00 & 166 & 36.75 & 63.25 & 256 & 98.05 & 1.95 & 918 \\
\hline Sardinia & 79 & 84.81 & 15.19 & 81 & $18: 52$ & 81.48 & 197 & 100.00 & 0.00 & 729 \\
\hline S Tyrrhenian Sea-Sicily Strait & 114 & 79.82 & 20.18 & 137 & 37.96 & 62.04 & 145 & 99.31 & 0.69 & 846 \\
\hline E Sicily-N Ionian Sea-SW Adriatic & 76 & 84.21 & 15.79 & 33 & $21: 21$ & 78.79 & 100 & 98.00 & 2.00 & 876 \\
\hline NW and Central Adriatic Sea & 29 & 79.31 & 20.69 & 13 & 61.54 & 38.46 & 121 & 99.17 & 0.83 & 515 \\
\hline Morocco & 14 & 64.29 & 35.71 & 8 & 25.00 & 75.00 & 6 & 83.33 & 16.67 & 63 \\
\hline N Adriatic-Slovenia & & & & & & & 9 & 100.00 & 0.00 & 0 \\
\hline TOTAL & 986 & 82.25 & 17.75 & 870 & 36.67 & 63.33 & 1420 & 98.24 & 1.76 & 6336 \\
\hline
\end{tabular}

macro-stratum (shelf/slope). Mean length values have been estimated for every geographical sector, depth macro-stratum and year.

In this paper the data from each geographical sector and depth stratum are reported. The distribution patterns of the abundance indices and mean lengths were analysed at the shelf (depth $<200 \mathrm{~m}$ ) and slope (depths between 200 and $800 \mathrm{~m}$ ) levels for each geographical sector.

In order to detect any temporal tendency, the biomass and density indices exceeding arbitrary threshold values of $5 \mathrm{~kg} \cdot \mathrm{km}^{-2}$ and 100 individuals $\cdot \mathrm{km}^{-2}$, respectively, were analysed through a linear regression of the log-transformed data $[\ln (x+1)]$ versus year, assuming a log-normal distribution of the trawl survey catches (Grosslein, 1971). The correlation coefficients were statistically tested. The mean lengths were compared among the geographical sectors after averaging the weighted yearly means in each sector. The presence of a linear trend from west to east was then analysed and the coefficients of correlation statistically tested.

\section{RESULTS}

Along the European coasts of the Mediterranean Sea, from the Straits of Gibraltar to the Aegean Sea (extended to Morocco since 1999), a total of 6,336 hauls was carried out on board several vessels. A total of 986, 870 and 1,420 occurrences of Pagellus acarne, Pagellus bogaraveo and Pagellus erythrinus were, respectively, analysed (Table 1).
On the whole, $P$. erythrinus was the most frequent species (Table 1), with a percentage of occurrence on the shelf close to $100 \%$. P. acarne also exhibited a similar pattern, although occurring with a lower frequency. Conversely, the tows giving a higher presence of the less common species, P. bogaraveo, were mainly located on the slope.

\section{Pagellus erythrinus}

This species was predominantly distributed in the two shallower strata $(10-100 \mathrm{~m})$, although it showed remarkable biomass and density indices at depths from 100 to $200 \mathrm{~m}$, mainly in North East and South East Corsica waters (Tables 2 and 3). The highest values were generally recorded in Argosaronikos at depths shallower than $50 \mathrm{~m}$. Considering the shelf, P. erythrinus was more abundant in Corsica waters, East Ionian, Argosaronikos and South Aegean Sea. In these areas, the biomass indices were generally greater than $20 \mathrm{~kg} \cdot \mathrm{km}^{-2}$, reaching the maximum value of $58.5 \mathrm{~kg} \cdot \mathrm{km}^{-2}(\mathrm{CV} \%=50.5)$ in Corsica waters in 1999. A comparable value was also observed in Argosaronikos in 1995. In the North Aegean Sea the biomass increased in the last three years, from $13.4(\mathrm{CV} \%=87.6)$ to $24.9 \mathrm{~kg} \cdot \mathrm{km}^{-2}$ $(\mathrm{CV} \%=84.8)$. Low values were conversely observed in the western sectors, from the Alborán Sea to the Gulf of Lions. The density indices showed a pattern analogous to that of biomass, reaching the maximum of 1,212 individuals $\cdot \mathrm{km}^{-2}$ $(\mathrm{CV} \%=33.2)$ in the East Ionian Sea in 1996.

Figure 1 shows the mean lengths per geographical sector and year with the corresponding 
TABLE 2. - Pagellus erythrinus: mean biomass $\left(\mathrm{kg} / \mathrm{km}^{2}\right)$ estimated from the MEDITS trawl surveys by depth stratum, geographical sector and year (1994-1999). Strata that were not sampled are indicated by '*'. Values higher than $10 \mathrm{~kg} / \mathrm{km}^{2}$ are presented in bold.

\begin{tabular}{|c|c|c|c|c|c|c|c|c|c|c|c|c|c|c|c|c|}
\hline \multirow{3}{*}{$\begin{array}{l}\text { Sector code } \\
111 \mathrm{a}\end{array}$} & \multirow{3}{*}{$\begin{array}{l}\text { Sector } \\
\text { Alborán Sea }\end{array}$} & \multicolumn{5}{|c|}{$\begin{array}{c}1994 \\
\text { Depth (m) }\end{array}$} & \multicolumn{5}{|c|}{$\begin{array}{c}1995 \\
\text { Depth (m) }\end{array}$} & \multicolumn{5}{|c|}{$\begin{array}{c}1996 \\
\text { Depth (m) }\end{array}$} \\
\hline & & $10-50$ & \multicolumn{3}{|c|}{$50-100$ 100-200 200-500 } & $500-800$ & \multicolumn{5}{|c|}{$\begin{array}{c}\text { Depth (m) } \\
10-50 \quad 50-100 \quad 100-200 \text { 200-500 500-800 }\end{array}$} & $10-50$ & $50-100$ & $100-200$ & :00-500 5 & :00-800 \\
\hline & & 43.3 & 8.3 & 0 & 0 & 0 & 0 & 34.3 & 0.9 & 0 & 0 & 1.1 & 2.6 & 0 & 0 & 0 \\
\hline $112 \mathrm{a}$ & Alicante & 0 & 0.4 & 0 & 0 & 0 & 2.2 & 1.7 & 0 & 0 & 0 & 1.5 & 4.9 & 0 & 0 & 0 \\
\hline $113 \mathrm{a}$ & Catalan Sea & 6.0 & 4.2 & 0 & 0 & 0 & 4.5 & 2.5 & 0.2 & 0 & 0 & 8.6 & 6.1 & 0 & 0 & 0 \\
\hline $114 a$ & W Morocco & * & $*$ & * & * & * & * & * & * & * & $*$ & $*$ & * & * & $*$ & * \\
\hline $114 \mathrm{~b}$ & E Morocco & * & $*$ & * & * & $*$ & * & * & $*$ & * & $*$ & $*$ & $*$ & * & $*$ & * \\
\hline $121 \mathrm{a}$ & W Gulf of Lions & 2.1 & 2.4 & 1.1 & 0 & 0 & 0.7 & 0.1 & 0 & 0 & 0 & 3.6 & 0.2 & 0 & 0 & 0 \\
\hline $121 \mathrm{~b}$ & E Gulf of Lions & 5.2 & 5.4 & 0 & 0 & 0 & 3.5 & 0 & 0 & 0 & 0 & 6.9 & 0 & 2.6 & 0 & 0 \\
\hline $131 \mathrm{a}$ & NE Corsica & * & 21.0 & 68.3 & 0 & 0 & * & 28.9 & 74.0 & 0 & 0 & $*$ & 46.2 & 32.9 & 1.1 & 0 \\
\hline $131 b$ & SE Corsica & * & 36.8 & 10.3 & 0 & 0 & * & 24.1 & 30.4 & 0 & 0 & * & 52.7 & 31.2 & 0 & 0 \\
\hline $132 \mathrm{a}$ & N Ligurian Sea & 2.5 & 12.7 & 0 & 0 & 0 & 11.7 & 0.6 & 1.8 & 0 & 0 & 12.1 & 3.1 & 0 & 0 & 0 \\
\hline $132 b$ & E Ligurian Sea & 22.7 & 11.7 & 7.9 & 0 & 0 & 9.6 & 7.0 & 3.6 & 0 & 0 & 41.6 & 7.8 & 2.1 & 0 & 0 \\
\hline $132 \mathrm{c}$ & N Tyrrhenian & 32.0 & 1.1 & 0 & 0 & 0 & 56.3 & 4.1 & 0 & 0 & 0 & 51.4 & 6.3 & 0 & 0 & 0 \\
\hline $132 \mathrm{~d}$ & C Tyrrhenian & 9.1 & 9.1 & 1.2 & 0 & 0 & 8.3 & 1.1 & 3.5 & 0 & 0 & 7.9 & 0.6 & 1.6 & 0 & 0 \\
\hline $133 \mathrm{a}$ & SE Sardinia & 4.7 & 11.6 & 0 & 0 & 0 & 7.2 & 2.3 & 0 & 0 & 0 & 4.4 & 5.4 & 0 & 0 & 0 \\
\hline $133 b$ & NE Sardinia & 0 & 3.5 & 8.7 & 0 & 0 & 0 & 3.1 & 0 & 0 & 0 & 8.4 & 14.2 & 4.3 & 0 & 0 \\
\hline $133 \mathrm{c}$ & N Sardinia & 5.9 & 8.5 & 0 & 0 & 0 & 5.2 & 1.1 & 0 & 0 & 0 & 5.9 & 12.1 & 0 & 0 & 0 \\
\hline $133 d$ & NW Sardinia & 28.6 & 37.3 & 0 & 0 & 0 & * & 19.3 & 4.9 & 0 & 0 & 34.8 & 8.4 & 2.0 & 0 & 0 \\
\hline $133 \mathrm{e}$ & W Sardinia & 28.1 & * & 1.4 & 0 & 0 & 14.3 & 7.8 & 0 & 0 & 0 & 39.3 & 2.3 & 0 & 0 & 0 \\
\hline $133 \mathrm{f}$ & SW Sardinia & 1.0 & 3.2 & 0 & 0 & 0 & 9.5 & 1.5 & 0 & 0 & 0 & 7.5 & 26.7 & 0 & 0 & 0 \\
\hline $133 \mathrm{~g}$ & S Sardinia & 7.8 & 0 & 0 & 0 & 0 & 12.6 & * & 0 & 0 & 0 & 1.2 & 2.1 & 0 & 0 & 0 \\
\hline $134 a$ & SE Tyrrhenian & 11.1 & 0.6 & 0.2 & 0 & 0 & 12.9 & 0.4 & 0 & 0 & 0 & 13.3 & 0.4 & 0.8 & 0 & 0 \\
\hline $134 \mathrm{~b}$ & SW Tyrrhenian & 6.2 & 63.8 & 5.7 & 0 & 0 & 24.3 & 24.7 & 15.3 & 0 & 0 & 65.9 & 48.3 & 0 & 0 & 0 \\
\hline $134 \mathrm{c}$ & Sicilian Chan. & 1.4 & 5.0 & 0 & 0 & 0 & 4.9 & 0.9 & 1.5 & 0 & 0 & 3.4 & 5.2 & 0.1 & 0 & 0 \\
\hline $211 \mathrm{a}$ & N Adriatic Sea & 0.3 & 1.5 & * & * & $*$ & 0.3 & 1.5 & * & $*$ & * & 0.4 & 0.9 & * & $*$ & * \\
\hline $211 b$ & Central Adriatic & 0.6 & 2.7 & 0 & 0 & 0 & 0.4 & 0 & 0 & 0 & 0 & 1.3 & 2.6 & 0 & 0 & 0 \\
\hline $211 \mathrm{c}$ & N Adriatic-Slov & $*$ & * & $*$ & * & $*$ & 136.4 & * & $*$ & $*$ & $*$ & 14.1 & * & $*$ & $*$ & * \\
\hline $211 d$ & NE Adri Croatia & * & * & * & * & $*$ & * & * & * & * & $*$ & 12.2 & 19.9 & 0.2 & 0 & * \\
\hline $221 \mathrm{a}$ & E Sicily & 3.5 & 0 & 0 & 0 & 0 & 5.7 & 2.9 & 1.9 & 0 & 0 & 11.7 & 5.2 & 5.4 & 0 & 0 \\
\hline $221 b$ & NW Ionian Sea & 0 & 0 & 0 & 0 & 0 & 0 & 4.4 & 2.7 & 0 & 0 & 0 & 0.0 & 3.5 & 0 & 0 \\
\hline $221 \mathrm{c}$ & N Ionian Sea & 2.0 & 0 & 0 & 0 & 0 & 1.0 & 0 & 0 & 0 & 0 & 0 & 1.3 & 0 & 0 & 0 \\
\hline $221 d$ & N Ionian Sea & $*$ & 0 & 0 & 0 & 0 & 1.9 & 0 & 0 & 0 & 0 & 0 & 1.9 & 0 & 0 & 0 \\
\hline $221 \mathrm{e}$ & SW Adriatic & 0 & 0 & 0 & 0 & 0 & * & 0 & 0 & 0 & 0 & * & 0 & 0 & 0 & 0 \\
\hline $221 \mathrm{f}$ & SW Adriatic & 0.5 & 0 & 0 & 0 & 0 & 1.2 & 0 & 0 & 0 & 0 & 0 & 0 & 0 & 0 & 0 \\
\hline $221 \mathrm{~g}$ & SW Adriatic & 0 & 0 & 0 & * & 0 & 0 & 0 & 0 & * & 0 & 0 & 0 & 0 & * & 0 \\
\hline $221 \mathrm{~h}$ & SW Adriatic & 0 & 0 & 0 & 0 & 0 & 0.1 & 0 & 0 & 0 & 0 & 0.1 & 0 & 0 & 0 & 0 \\
\hline $221 \mathrm{i}$ & SE Adriatic & * & * & * & * & $*$ & * & * & * & * & $*$ & 15.7 & 5.5 & 0 & 0 & 0 \\
\hline $222 \mathrm{a}$ & E Ionian Sea & 0 & 54.2 & 0 & 0 & 0 & 6.2 & 28.0 & 0 & 0 & 0 & 81.8 & 34.0 & 0 & 0 & 0 \\
\hline $223 \mathrm{a}$ & Argosaronikos & 109.4 & 19.1 & 0 & 0 & 0 & 226.2 & 7.6 & 0 & 0 & 0 & 114.2 & 69.9 & 4.5 & 0 & 0 \\
\hline $224 a$ & N Aegean Sea & 1.2 & 1.9 & 5.4 & 0 & 0 & 21.4 & 18.6 & 0.2 & 0 & 0 & 21.9 & 0.3 & 2.8 & 0 & 0 \\
\hline $225 \mathrm{a}$ & S Aegean Sea & 13.9 & 21.1 & 0 & 0 & 0 & 83.6 & 18.8 & 11.2 & 0 & 0 & 13.8 & 53.3 & 4.8 & 0 & 0 \\
\hline & & & & 1997 & & & & & $\begin{array}{r}1998 \\
\text { Depth }\end{array}$ & & & & & $\begin{array}{r}1999 \\
\text { Depth (1 }\end{array}$ & & \\
\hline Sector code & Sector & $10-50$ & $50-100$ & $00-200$ & $00-500$ & 500-800 & $10-50$ & $50-100$ & $100-200$ & $00-500$ & $00-800$ & $10-50$ & $50-100$ & & $00-500=$ & \\
\hline $111 \mathrm{a}$ & Alborán Sea & 27.9 & 3.2 & 0 & 0 & 0 & 0 & 9.1 & 0 & 0 & 0 & 0 & 4.8 & 0 & 0 & 0 \\
\hline $112 \mathrm{a}$ & Alicante & 1.3 & 1.3 & 0 & 0 & 0 & 1.9 & 4.4 & 0 & 0 & 0 & 22.3 & 11.9 & 0 & 0 & 0 \\
\hline $113 \mathrm{a}$ & Catalan Sea & 22.2 & 5.2 & 0 & 0 & 0 & 9.8 & 9.1 & 1.0 & $*$ & 0 & 35.0 & 12.5 & 0.8 & 0 & 0 \\
\hline $114 a$ & W Morocco & $*$ & $*$ & $*$ & * & $*$ & $*$ & * & * & $*$ & $*$ & $*$ & 3.8 & 0 & 0 & 1.5 \\
\hline $114 \mathrm{~b}$ & E Morocco & * & $*$ & * & * & * & * & * & * & * & $*$ & 18.1 & 1.7 & 0 & 0 & 0 \\
\hline $121 \mathrm{a}$ & W Gulf of Lions & 2.5 & 0 & 0 & 0 & 0 & 7.9 & 0.3 & 0 & 0 & 0 & 6.8 & 1.2 & 0 & 0 & 0 \\
\hline $121 \mathrm{~b}$ & E Gulf of Lions & 0.4 & 3.3 & 0 & 0 & 0 & 6.1 & 4.7 & 0 & 0 & 0 & 41.3 & 3.5 & 0 & 0 & * \\
\hline $131 \mathrm{a}$ & NE Corsica & * & 26.7 & * & 2.2 & 0 & $*$ & 22.3 & 33.5 & 0.3 & 0 & * & 40.8 & 18.4 & 3.2 & 0 \\
\hline $131 b$ & SE Corsica & $*$ & 13.8 & 0 & 0 & $*$ & $*$ & 18.8 & 10.8 & 0 & 0 & $*$ & 110.6 & 1.2 & 0 & 0 \\
\hline $132 \mathrm{a}$ & N Ligurian Sea & 31.2 & 2.4 & 0 & 0 & 0 & 29.1 & 49.8 & 0.5 & 0 & 0 & 36.4 & 1.6 & 0 & 0 & 0 \\
\hline $132 b$ & E Ligurian Sea & 27.1 & 4.4 & 2.6 & 0 & 0 & 24.8 & 9.7 & 3.5 & 0.1 & 0 & 44.8 & 12.8 & 4.4 & 0 & 0 \\
\hline $132 \mathrm{c}$ & N Tyrrhenian & 29.0 & 0.4 & 0.2 & 0 & 0 & 25.9 & 0.5 & 0 & 0 & 0 & 40.3 & 5.4 & 0 & 0 & 0 \\
\hline $132 \mathrm{~d}$ & C Tyrrhenian & 8.2 & 3.7 & 1.4 & 0 & 0 & 12.1 & 8.0 & 1.1 & 0 & 0 & 23.6 & 4.9 & 2.4 & 0.5 & 0 \\
\hline $133 a$ & SE Sardinia & 2.2 & 11.4 & 0 & 0 & 0 & 5.4 & 16.2 & 0 & 0 & 0 & 7.7 & 3.8 & 0 & 0 & 0 \\
\hline $133 b$ & NE Sardinia & 7.7 & 13.0 & 0 & 0 & 0 & 16.7 & 11.4 & 8.9 & 0 & 0 & 2.9 & 7.6 & 5.3 & 0 & 0 \\
\hline $133 \mathrm{c}$ & N Sardinia & 3.0 & 10.6 & 0 & 0 & 0 & 22.9 & 11.5 & 0 & 0 & 0 & 25.7 & 12.2 & 0 & 0 & 0 \\
\hline $133 d$ & NW Sardinia & 26.0 & 17.7 & 0 & 0 & 0 & 27.3 & 18.2 & 0 & 0 & 0 & 32.1 & 25.6 & 0.6 & 0 & 0 \\
\hline $133 \mathrm{e}$ & W Sardinia & 2.9 & 19.4 & 0 & 0 & 0 & 17.6 & 5.4 & 0 & 0 & 0 & 22.4 & 20.8 & 0 & 0 & 0 \\
\hline $133 \mathrm{f}$ & SW Sardinia & 2.6 & 18.4 & 0 & 0 & 0 & 17.5 & 6.1 & 0 & 0 & 0 & 9.1 & 20.9 & 0.3 & 0 & 0 \\
\hline $133 \mathrm{~g}$ & S Sardinia & 1.1 & 1.1 & 0 & 0 & 0 & 5.0 & 0 & 2.3 & 0 & 0 & 4.1 & 7.1 & 0 & 0 & 0 \\
\hline $134 \mathrm{a}$ & SE Tyrrhenian & 7.5 & 6.8 & 0.7 & 0 & 0 & 26.8 & 4.1 & 0 & 0 & 0 & 14.9 & 17.3 & 0.1 & 0 & 0 \\
\hline $134 \mathrm{~b}$ & SW Tyrrhenian & 55.8 & 71.4 & 0 & 0 & 0 & 25.0 & 31.7 & 5.7 & 0 & 0 & 26.7 & 36.6 & 2.7 & 0 & 0 \\
\hline
\end{tabular}


TABLE 2 (Cont.). - Pagellus erythrinus: mean biomass $\left(\mathrm{kg} / \mathrm{km}^{2}\right)$ estimated from the MEDITS trawl surveys by depth stratum, geographical sector and year (1994-1999). Strata that were not sampled are indicated by '*'. Values higher than $10 \mathrm{~kg} / \mathrm{km}^{2}$ are presented in bold.

\begin{tabular}{|c|c|c|c|c|c|c|c|c|c|c|c|c|c|c|c|c|}
\hline \multirow{2}{*}{$\begin{array}{l}\text { Sector code } \\
134 \mathrm{c}\end{array}$} & \multirow{2}{*}{$\begin{array}{l}\text { Sector } \\
\text { Sicilian Chan. }\end{array}$} & \multicolumn{5}{|c|}{\begin{tabular}{ccc}
\multicolumn{1}{c}{1997} \\
\multicolumn{1}{c}{ Depth (m) } \\
$10-50 \quad 50-100$ 100-200 200-500 $500-800$
\end{tabular}} & \multicolumn{5}{|c|}{$\begin{array}{c}1998 \\
\text { Depth (m) }\end{array}$} & \multicolumn{5}{|c|}{$\begin{array}{ccc} & 1999 \\
& \text { Depth (m) } & \\
10-50 & 50-100 \quad & 100-200 \\
200-500 & 500-800\end{array}$} \\
\hline & & 1.6 & 8.2 & 0.4 & 0 & 0 & 2.8 & 27.0 & 0.1 & 0 & 0 & 3.4 & 7.5 & 0.2 & 0 & 0 \\
\hline $211 \mathrm{a}$ & N Adriatic Sea & 0.8 & 0.9 & * & * & * & 0.4 & 0.5 & * & * & * & 7.1 & 1.1 & * & * & * \\
\hline $211 b$ & Central Adriatic & 1.8 & 1.9 & 0 & 0 & 0 & 0.4 & 0.4 & 0 & 0.1 & $*$ & 3.0 & 0.6 & 0 & 0 & * \\
\hline $211 \mathrm{c}$ & N Adriatic-Slov & 37.9 & * & * & * & * & 52.2 & * & * & * & $*$ & 56.0 & * & * & $*$ & * \\
\hline $211 d$ & NE Adri Croatia & 10.5 & 35.6 & 2.5 & 0 & $*$ & 32.6 & 14.7 & 1.8 & 0 & $*$ & 0 & $*$ & $*$ & $*$ & * \\
\hline $221 \mathrm{a}$ & E Sicily & 17.2 & 7.4 & 0 & 0 & 0 & 36.5 & 6.7 & 4.6 & 0 & 0 & 28.2 & 0.4 & 0 & 0 & 0 \\
\hline $221 b$ & NW Ionian Sea & 0 & 5.4 & 0.3 & 0 & 0 & 2.0 & 12.9 & 1.1 & 0 & 0.1 & 5.4 & 0 & 0 & 0 & 0 \\
\hline $221 \mathrm{c}$ & N Ionian Sea & 11.1 & 0 & 0 & 0 & 0 & 2.1 & 6.0 & 0 & 0 & 0 & 2.3 & 3.2 & 0 & 0 & 0 \\
\hline $221 d$ & N Ionian Sea & 6.5 & 0 & 0 & 0 & 0 & 3.3 & 1.0 & 0 & 0 & 0 & 30.4 & 0 & 0 & 0 & 0 \\
\hline $221 \mathrm{e}$ & SW Adriatic & $*$ & 0 & 0 & 0 & 0 & * & 0 & 0 & 0 & 0 & $*$ & 0 & 0.2 & 0 & 0 \\
\hline $221 \mathrm{f}$ & SW Adriatic & 1.0 & 0.1 & 0 & 0 & 0 & 8.3 & 0.1 & 0.3 & 0 & 0 & 5.2 & 0 & 0 & 0 & 0 \\
\hline $221 \mathrm{~g}$ & SW Adriatic & 0.8 & 0 & 0 & * & 0 & 1.6 & 0.2 & 0 & * & 0 & 3.0 & 0 & 0 & * & 0 \\
\hline $221 \mathrm{~h}$ & SW Adriatic & 0.2 & 0.5 & 0 & 0 & 0 & 0.4 & 0 & 0 & 0 & 0 & 2.5 & 0.4 & 1.0 & 1.3 & 0 \\
\hline $221 \mathrm{i}$ & SE Adriatic & 18.9 & 3.6 & 0 & 0 & 0 & 10.4 & 6.5 & 0 & 0.1 & 0 & 71.6 & 16.4 & 0 & 0 & 0 \\
\hline $222 a$ & E Ionian Sea & 30.1 & 26.7 & 0 & 0 & 0 & 75.4 & 14.8 & 1.2 & 0 & 0 & 41.3 & 16.0 & 1.7 & 0 & 0 \\
\hline $223 a$ & Argosaronikos & 163.5 & 44.1 & 1.8 & 0 & 0 & 72.0 & 51.7 & 3.9 & 0 & 0 & 71.0 & 82.5 & 18.5 & 0 & 0 \\
\hline $224 a$ & N Äegean Sea & 89.1 & 0.3 & 3.0 & 0 & 0 & 51.3 & 0.2 & 2.0 & 0 & 0 & 48.1 & 0.8 & 1.2 & 0 & 0 \\
\hline $225 \mathrm{a}$ & S Aegean Sea & 14.4 & 20.8 & 9.4 & 0.1 & 0 & 134.8 & 28.9 & 5.8 & 0.1 & 0 & 60.5 & 25.3 & 11.4 & 0.3 & 0 \\
\hline
\end{tabular}

TABLE 3. - Pagellus erythrinus: mean density (in number of individuals $/ \mathrm{km}^{2}$ ) estimated from the MEDITS trawl surveys by depth stratum, geographical sector and year (1994-1999). Strata that were not sampled are indicated by '*'. Values higher than 200 individuals $/ \mathrm{km}^{2}$ are presented in bold.

\begin{tabular}{|c|c|c|c|c|c|c|c|c|c|c|c|c|c|c|c|c|}
\hline \multirow{2}{*}{$\begin{array}{l}\text { Sector code } \\
111 \mathrm{a}\end{array}$} & \multirow{2}{*}{$\begin{array}{l}\text { Sector } \\
\text { Alborán Sea }\end{array}$} & \multicolumn{5}{|c|}{$\begin{array}{c}1994 \\
\text { Depth (m) }\end{array}$} & \multicolumn{5}{|c|}{$\begin{array}{c}1995 \\
\text { Depth (m) }\end{array}$} & \multicolumn{5}{|c|}{$\begin{array}{c}1996 \\
\text { Depth (m) }\end{array}$} \\
\hline & & 163 & 30 & 0 & 0 & 0 & 0 & 151 & 6 & 0 & 0 & 11 & 31 & 0 & 0 & 0 \\
\hline $112 \mathrm{a}$ & Alicante & 0 & 4 & 0 & 0 & 0 & 27 & 14 & 0 & 0 & 0 & 16 & 35 & 0 & 0 & 0 \\
\hline $113 a$ & Catalan Sea & 123 & 36 & 0 & 0 & 0 & 78 & 23 & 2 & 0 & 0 & 99 & 52 & 0 & 0 & 0 \\
\hline $114 \mathrm{a}$ & W Morocco & * & $*$ & * & $*$ & $*$ & * & * & * & $*$ & $*$ & * & * & $*$ & * & * \\
\hline $114 \mathrm{~b}$ & E Morocco & $*$ & $*$ & $*$ & $*$ & $*$ & $*$ & $*$ & $*$ & $*$ & $*$ & $*$ & $*$ & $*$ & $*$ & $*$ \\
\hline $121 \mathrm{a}$ & W Gulf of Lions & 17 & 8 & 10 & 0 & 0 & 3 & 1 & 0 & 0 & 0 & 27 & 1 & 0 & 0 & 0 \\
\hline $121 \mathrm{~b}$ & E Gulf of Lions & 54 & 19 & 0 & 0 & 0 & 7 & 0 & 0 & 0 & 0 & 54 & 0 & 10 & 0 & 0 \\
\hline $131 \mathrm{a}$ & NE Corsica & * & 490 & 863 & 0 & 0 & * & 685 & 779 & 0 & 0 & $*$ & 873 & 473 & 7 & 0 \\
\hline $131 \mathrm{~b}$ & SE Corsica & $*$ & 743 & 98 & 0 & 0 & * & 415 & 336 & 0 & 0 & $*$ & 1049 & 479 & 0 & 0 \\
\hline $132 \mathrm{a}$ & N Ligurian Sea & 77 & 61 & 0 & 2 & 0 & 220 & 7 & 38 & 0 & 0 & 208 & 54 & 0 & 0 & 0 \\
\hline $132 b$ & E Ligurian Sea & 332 & 159 & 49 & 0 & 0 & 189 & 127 & 38 & 0 & 0 & 878 & 97 & 20 & 0 & 0 \\
\hline $132 \mathrm{c}$ & N Tyrrhenian & 952 & 18 & 0 & 1 & 0 & 2235 & 45 & 0 & 0 & 0 & 1505 & 80 & 0 & 0 & 0 \\
\hline $132 d$ & C Tyrrhenian & 123 & 95 & 9 & 0 & 0 & 151 & 13 & 20 & 0 & 0 & 111 & 7 & 14 & 0 & 0 \\
\hline $133 \mathrm{a}$ & SE Sardinia & 186 & 95 & 0 & 0 & 0 & 262 & 53 & 0 & 0 & 0 & 80 & 122 & 0 & 0 & 0 \\
\hline $133 b$ & NE Sardinia & 0 & 41 & 54 & 0 & 0 & 0 & 48 & 0 & 0 & 0 & 97 & 185 & 43 & 0 & 0 \\
\hline $133 \mathrm{c}$ & N Sardinia & 104 & 73 & 0 & 0 & 0 & 127 & 11 & 0 & 0 & 0 & 120 & 106 & 0 & 0 & 0 \\
\hline $133 d$ & NW Sardinia & 246 & 403 & 0 & 0 & 0 & $*$ & 215 & 43 & 0 & 0 & 342 & 59 & 58 & 0 & 0 \\
\hline $133 \mathrm{e}$ & W Sardinia & 581 & $*$ & 12 & 0 & 0 & 497 & 90 & 0 & 0 & 0 & 896 & 13 & 0 & 0 & 0 \\
\hline $133 \mathrm{f}$ & SW Sardinia & 21 & 37 & 0 & 0 & 0 & 432 & 29 & 0 & 0 & 0 & 62 & 251 & 0 & 0 & 0 \\
\hline $133 \mathrm{~g}$ & S Sardinia & 371 & 0 & 0 & 0 & 0 & 317 & $*$ & 0 & 0 & 0 & 12 & 11 & 0 & 0 & 0 \\
\hline $134 \mathrm{a}$ & SE Tyrrhenian & 180 & 11 & 2 & 0 & 0 & 324 & 7 & 0 & 0 & 0 & 267 & 7 & 9 & 0 & 0 \\
\hline $134 b$ & SW Tyrrhenian & 140 & 777 & 67 & 0 & 0 & 412 & 232 & 140 & 0 & 0 & 626 & 376 & 0 & 0 & 0 \\
\hline $134 \mathrm{c}$ & Sicilian Chan. & 36 & 32 & 0 & 0 & 0 & 140 & 24 & 3 & 0 & 0 & 92 & 73 & 2 & 0 & 0 \\
\hline $211 \mathrm{a}$ & N Adriatic Sea & 16 & 24 & * & * & $*$ & 9 & 29 & * & * & $*$ & 12 & 17 & * & * & $*$ \\
\hline $211 \mathrm{~b}$ & Central Adriatic & 11 & 22 & 0 & 0 & 0 & 10 & 0 & 0 & 0 & 0 & 17 & 16 & 0 & 0 & 0 \\
\hline $211 \mathrm{c}$ & N Adriatic-Slov & * & * & * & * & $*$ & 1307 & * & * & * & $*$ & 94 & $*$ & * & * & * \\
\hline $211 d$ & NE Adri Croatia & * & * & * & * & $*$ & $*$ & * & * & * & $*$ & 279 & 388 & 1 & 0 & * \\
\hline $221 \mathrm{a}$ & E Sicily & 71 & 0 & 0 & 0 & 0 & 105 & 44 & 21 & 0 & 0 & 263 & 31 & 40 & 0 & 0 \\
\hline $221 b$ & NW Ionian Sea & 0 & 0 & 0 & 0 & 0 & 0 & 22 & 11 & 0 & 0 & 0 & 0 & 12 & 0 & 0 \\
\hline $221 \mathrm{c}$ & N Ionian Sea & 31 & 0 & 0 & 0 & 0 & 26 & 0 & 0 & 0 & 0 & 0 & 14 & 0 & 0 & 0 \\
\hline $221 d$ & N Ionian Sea & * & 0 & 0 & 0 & 0 & 42 & 0 & 0 & 0 & 0 & 0 & 19 & 0 & 0 & 0 \\
\hline $221 \mathrm{e}$ & SW Adriatic & 0 & 0 & 0 & 0 & 0 & * & 0 & 0 & 0 & 0 & $*$ & 0 & 0 & 0 & 0 \\
\hline $221 \mathrm{f}$ & SW Adriatic & 7 & 0 & 0 & 0 & 0 & 75 & 0 & 0 & 0 & 0 & 0 & 0 & 0 & 0 & 0 \\
\hline $221 \mathrm{~g}$ & SW Adriatic & 0 & 0 & 0 & * & 0 & 0 & 0 & 0 & * & 0 & 0 & 0 & 0 & * & 0 \\
\hline $221 \mathrm{~h}$ & SW Adriatic & 0 & 0 & 0 & 0 & 0 & 8 & 0 & 0 & 0 & 0 & 8 & 0 & 0 & 0 & 0 \\
\hline $221 i$ & SE Adriatic & * & * & $*$ & * & $*$ & * & $*$ & * & * & $*$ & 256 & 73 & 0 & 0 & 0 \\
\hline $222 \mathrm{a}$ & E Ionian Sea & 0 & 497 & 0 & 0 & 0 & 212 & 236 & 0 & 0 & 0 & 3268 & 543 & 0 & 0 & 0 \\
\hline $223 a$ & Argosaronikos & 2057 & 83 & 0 & 0 & 0 & 2692 & 68 & 0 & 0 & 0 & 2218 & 894 & 38 & 0 & 0 \\
\hline $224 a$ & N Aegean Sea & 10 & 19 & 16 & 0 & 0 & 71 & 113 & 2 & 0 & 0 & 355 & 4 & 26 & 0 & 0 \\
\hline $225 \mathrm{a}$ & S Aegean Sea & 253 & 125 & 0 & 0 & 0 & 1096 & 283 & 93 & 0 & 0 & 969 & 774 & 35 & 0 & 0 \\
\hline
\end{tabular}


TABLE 3 (Cont.). - Pagellus erythrinus: mean density (in number of individuals $/ \mathrm{km}^{2}$ ) estimated from the MEDITS trawl surveys by depth stratum, geographical sector and year (1994-1999). Strata that were not sampled are indicated by '*'. Values higher than 200 individuals /km ${ }^{2}$ are presented in bold.

\begin{tabular}{|c|c|c|c|c|c|c|c|c|c|c|c|c|c|c|c|c|}
\hline \multirow{2}{*}{$\begin{array}{l}\text { Sector code } \\
111 \mathrm{a}\end{array}$} & \multirow{2}{*}{$\begin{array}{l}\text { Sector } \\
\text { Alborán Sea }\end{array}$} & \multicolumn{5}{|c|}{$\begin{array}{c}1997 \\
\text { Depth (m) }\end{array}$} & \multicolumn{5}{|c|}{$\begin{array}{c}1998 \\
\text { Depth (m) }\end{array}$} & \multicolumn{5}{|c|}{$\begin{array}{c}1999 \\
\text { Depth (m) }\end{array}$} \\
\hline & & $\begin{array}{r}10-50 \\
\mathbf{2 3 1}\end{array}$ & 17 & 0 & 0 & 0 & $\begin{array}{r}10-50 \\
0\end{array}$ & $\begin{array}{r}50-100 \\
24\end{array}$ & 0 & 0 & 0 & $\begin{array}{r}10-50 \\
0\end{array}$ & $\begin{array}{r}50-100 \\
20\end{array}$ & 0 & 0 & 0 \\
\hline $112 \mathrm{a}$ & Alicante & 9 & 8 & 0 & 0 & 0 & 11 & 31 & 0 & 0 & 0 & 287 & 107 & 0 & 0 & 0 \\
\hline $113 \mathrm{a}$ & Catalan Sea & 217 & 44 & 0 & 0 & 0 & 231 & 70 & 6 & $*$ & 0 & 471 & 92 & 4 & 0 & 0 \\
\hline $114 \mathrm{a}$ & W Morocco & $*$ & $*$ & $*$ & $*$ & $*$ & $*$ & $*$ & $*$ & $*$ & $*$ & $*$ & 21 & 0 & 0 & 5 \\
\hline $114 b$ & E Morocco & $*$ & $*$ & $*$ & $*$ & $*$ & $*$ & $*$ & $*$ & $*$ & $*$ & 276 & 11 & 0 & 0 & 0 \\
\hline $121 \mathrm{a}$ & W Gulf of Lions & 14 & 0 & 0 & 0 & 0 & 133 & 3 & 0 & 0 & 0 & 48 & 10 & 0 & 0 & 0 \\
\hline $121 b$ & E Gulf of Lions & 3 & 17 & 0 & 0 & 0 & 151 & 83 & 0 & 0 & 0 & 524 & 20 & 0 & 0 & $*$ \\
\hline $131 \mathrm{a}$ & NE Corsica & $*$ & 282 & $*$ & 15 & 0 & $*$ & 534 & 512 & 6 & 0 & $*$ & 763 & 221 & 46 & 0 \\
\hline $131 b$ & SE Corsica & $*$ & 234 & 0 & 0 & $*$ & $*$ & 304 & 149 & 0 & 0 & $*$ & 1627 & 9 & 0 & 0 \\
\hline $132 \mathrm{a}$ & N Ligurian Sea & 1105 & 24 & 0 & 0 & 0 & 677 & 706 & 7 & 0 & 0 & 310 & 21 & 0 & 0 & 0 \\
\hline $132 b$ & E Ligurian Sea & 795 & 51 & 22 & 0 & 0 & 520 & 160 & 27 & 2 & 0 & 602 & 143 & 33 & 0 & 0 \\
\hline $132 \mathrm{c}$ & N Tyrrhenian & 683 & 7 & 2 & 0 & 0 & 659 & 7 & 0 & 0 & 0 & 569 & 73 & 2 & 0 & 0 \\
\hline $132 d$ & C Tyrrhenian & 2371 & 46 & 9 & 0 & 0 & 252 & 139 & 9 & 0 & 0 & 265 & 75 & 23 & 3 & 0 \\
\hline $133 \mathrm{a}$ & SE Sardinia & 87 & 119 & 0 & 0 & 0 & 123 & 336 & 0 & 0 & 0 & 759 & 45 & 0 & 0 & 0 \\
\hline $133 b$ & NE Sardinia & 107 & 164 & 0 & 0 & 0 & 390 & 134 & 83 & 0 & 0 & 34 & 114 & 32 & 0 & 0 \\
\hline $133 \mathrm{c}$ & N Sardinia & 45 & 141 & 0 & 0 & 0 & 317 & 104 & 0 & 0 & 0 & 359 & 115 & 0 & 0 & 0 \\
\hline $133 d$ & NW Sardinia & 215 & 180 & 0 & 0 & 0 & 297 & 245 & 0 & 0 & 0 & 222 & 333 & 8 & 0 & 0 \\
\hline $133 \mathrm{e}$ & W Sardinia & 29 & 223 & 0 & 0 & 0 & 317 & 41 & 0 & 0 & 0 & 592 & 156 & 0 & 0 & 0 \\
\hline $133 \mathrm{f}$ & SW Sardinia & 38 & 152 & 0 & 0 & 0 & 713 & 61 & 0 & 0 & 0 & 269 & 301 & 4 & 0 & 0 \\
\hline $133 \mathrm{~g}$ & S Sardinia & 39 & 11 & 0 & 0 & 0 & 173 & 0 & 18 & 0 & 0 & 94 & 60 & 0 & 0 & 0 \\
\hline $134 \mathrm{a}$ & SE Tyrrhenian & 147 & 93 & 7 & 0 & 0 & 503 & 82 & 0 & 0 & 0 & 298 & 250 & 2 & 0 & 0 \\
\hline $134 b$ & SW Tyrrhenian & 437 & 649 & 0 & 0 & 0 & 443 & 235 & 45 & 0 & 0 & 408 & 295 & 19 & 0 & 0 \\
\hline $134 \mathrm{c}$ & Sicilian Chan. & 45 & 93 & 4 & 0 & 0 & 73 & 200 & 2 & 0 & 0 & 57 & 77 & 4 & 0 & 0 \\
\hline $211 \mathrm{a}$ & N Adriatic Sea & 42 & 14 & $*$ & $*$ & $*$ & 24 & 9 & $*$ & $*$ & $*$ & 384 & 15 & $*$ & $*$ & $*$ \\
\hline $211 b$ & Central Adriatic & 84 & 23 & 0 & 0 & 0 & 5 & 6 & 1 & 1 & $*$ & 90 & 8 & 0 & 0 & $*$ \\
\hline $211 \mathrm{c}$ & N Adriatic-Slov & 360 & $*$ & $*$ & $*$ & $*$ & 315 & $*$ & $*$ & $*$ & $*$ & 778 & $*$ & $*$ & $*$ & $*$ \\
\hline $211 d$ & NE Adri Croatia & 237 & 577 & 13 & 0 & $*$ & 628 & 213 & 15 & 0 & $*$ & 0 & $*$ & $*$ & $*$ & $*$ \\
\hline $221 \mathrm{a}$ & E Sicily & 296 & 84 & 0 & 0 & 0 & 694 & 78 & 38 & 0 & 0 & 801 & 11 & 0 & 0 & 0 \\
\hline $221 b$ & NW Ionian Sea & 0 & 33 & 11 & 0 & 0 & 23 & 151 & 11 & 0 & 1 & 69 & 0 & 0 & 0 & 0 \\
\hline $221 \mathrm{c}$ & N Ionian Sea & 96 & 0 & 0 & 0 & 0 & 22 & 58 & 0 & 0 & 0 & 37 & 44 & 0 & 0 & 0 \\
\hline $221 d$ & N Ionian Sea & 65 & 0 & 0 & 0 & 0 & 44 & 22 & 0 & 0 & 0 & 1620 & 0 & 0 & 0 & 0 \\
\hline $221 \mathrm{e}$ & SW Adriatic & $*$ & 0 & 0 & 0 & 0 & $*$ & 0 & 0 & 0 & 0 & $*$ & 0 & 3 & 0 & 0 \\
\hline $221 f$ & SW Adriatic & 39 & 7 & 0 & 0 & 0 & 515 & 8 & 4 & 0 & 0 & 158 & 0 & 0 & 0 & 0 \\
\hline $221 \mathrm{~g}$ & SW Adriatic & 24 & 0 & 0 & $*$ & 0 & 54 & 15 & 0 & $*$ & 0 & 106 & 0 & 0 & $*$ & 0 \\
\hline $221 \mathrm{~h}$ & SW Adriatic & 3 & 4 & 0 & 0 & 0 & 13 & 4 & 0 & 0 & 0 & 108 & 15 & 19 & 26 & 0 \\
\hline $221 \mathrm{i}$ & SE Adriatic & 304 & 51 & 0 & 0 & 0 & 249 & 110 & 0 & 1 & 0 & 1886 & 376 & 0 & 0 & 0 \\
\hline $222 \mathrm{a}$ & E Ionian Sea & 557 & 351 & 0 & 0 & 0 & 1946 & 283 & 16 & 0 & 0 & 2206 & 316 & 18 & 2 & 0 \\
\hline $223 a$ & Argosaronikos & 3042 & 722 & 6 & 0 & 0 & 1540 & 563 & 21 & 0 & 0 & 1447 & 1166 & 76 & 0 & 0 \\
\hline $224 a$ & N Aegean Sea & 760 & 4 & 29 & 0 & 0 & 673 & 2 & 25 & 0 & 0 & 474 & 16 & 13 & 0 & 0 \\
\hline $225 a$ & S Aegean Sea & 272 & 259 & 105 & 1 & 0 & 2586 & 474 & 78 & 1 & 0 & 1261 & 522 & 127 & 4 & 0 \\
\hline
\end{tabular}

standard deviations. The data are only presented for the shelf $(19,900$ individuals $)$, since only 82 fish were caught on the slope. The largest absolute length of $38 \mathrm{~cm}$ was observed in the Alborán Sea and the smallest $(2 \mathrm{~cm})$ in LigurianNorth Central Tyrrhenian Sea. However, even in the western side, juveniles of $4 \mathrm{~cm}$ length occurred in the samples.

The smallest mean length $(7.8 \mathrm{~cm})$ was recorded in Ligurian-North Central Tyrrhenian Sea in 1997, while the largest $(26.0 \mathrm{~cm})$ was found in Alicante and in the Gulf of Lions in 1994 and 1995, respectively. The greater mean lengths were mainly found in the western sectors, from Alborán to the Gulf of Lions, but also in the North Aegean Sea. The mean lengths from west to east showed a highly significant $(\mathrm{p}<0.05)$ decreasing trend.

\section{Pagellus acarne}

This species was mainly distributed on the continental shelf, in the strata from 10 to $200 \mathrm{~m}$, although high biomass indices were also recorded from 200 to $500 \mathrm{~m}$, especially so in the East Gulf of Lions (Table 4), where, however, the corresponding densities (Table 5) were not as high, indicating the occurrence of larger specimens. The highest density was observed in the eastern part of Morocco in 1999 at the shallowest depths. A comparable value was also recorded in 1998 in the South Aegean Sea (Table 5). Considering the shelf, the catches recorded in the Alborán Sea dominated the scenario, with biomass values up to $406.3 \mathrm{~kg} \cdot \mathrm{km}^{-2}(\mathrm{CV} \%=37.0)$ in 1994. The corresponding density index was 2,898 individuals $\cdot \mathrm{km}^{-2}(\mathrm{CV} \%=27.7)$, a number considerably 

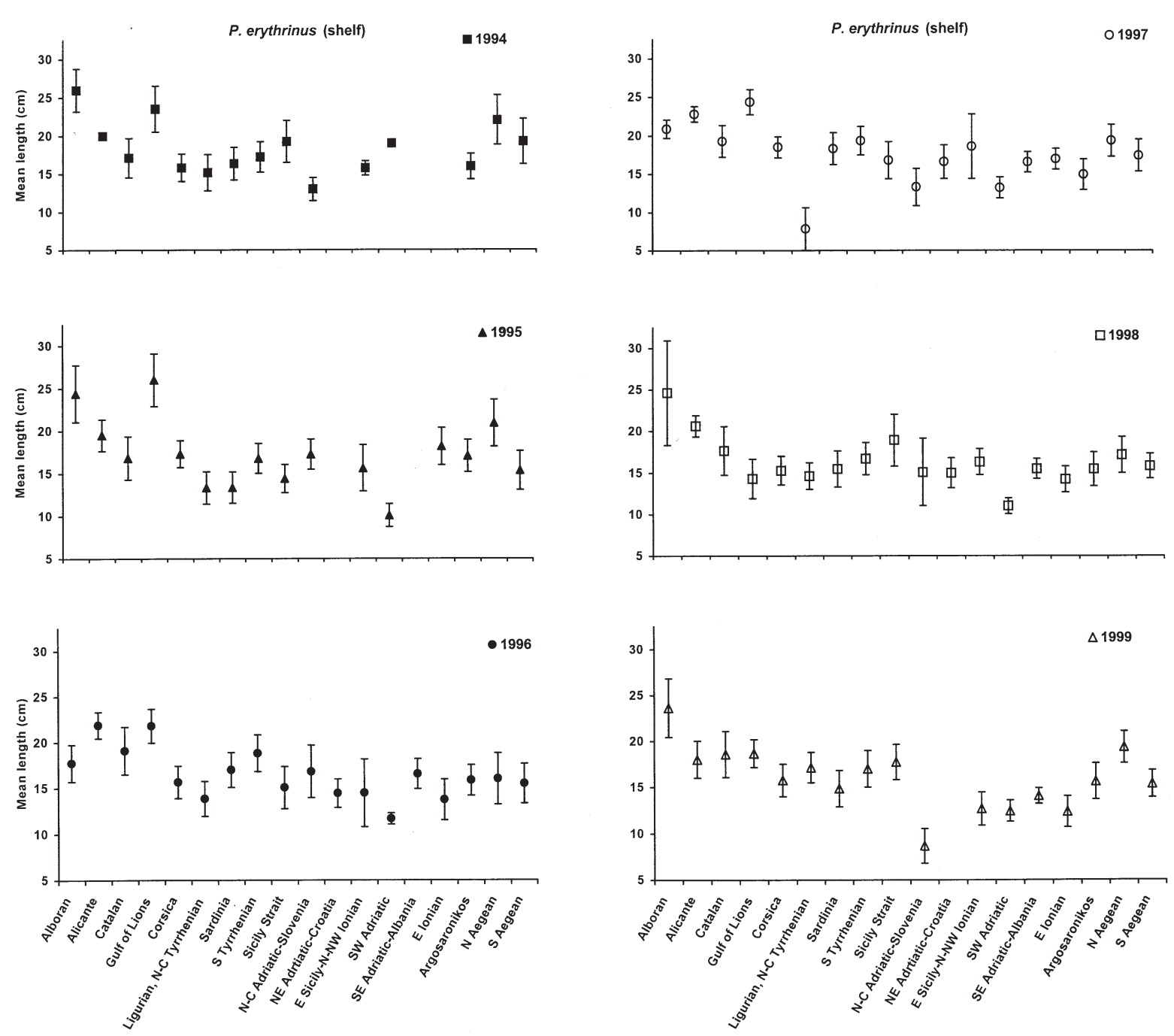

FIG. 1. - Mean total length ( \pm S.D.) of Pagellus erythrinus on the continental shelf by geographical region and year.

lower than that observed in 1998 in the South Aegean Sea, where the density reached the value of 5,084 individuals $\cdot \mathrm{km}^{-2}(\mathrm{CV} \%=78.6)$, but with a biomass of $75.0 \mathrm{~kg} \cdot \mathrm{km}^{-2}(\mathrm{CV} \%=73.8)$. This was due to the occurrence of smaller fishes in the easternmost area. $P$. acarne also showed a remarkable biomass $\left(146.3 \mathrm{~kg} \cdot \mathrm{km}^{-2}\right.$; $\left.\mathrm{CV} \%=38.5\right)$ and density $(6,579$ individuals $\cdot \mathrm{km}^{-2}(\mathrm{CV} \%=61.0)$ on the continental shelf of Morocco, where the trawl survey started in 1999. Catches on the slope were very poor and density was highly variable with less than 33 individuals $\mathrm{km}^{-2}$, except in the South Aegean Sea (76 individuals $\cdot \mathrm{km}^{-}$ 2. $\mathrm{CV} \%=96,5)$. The biomass indices were below 9.1 $\mathrm{kg} \cdot \mathrm{km}^{-2}(\mathrm{CV} \%=113.2)$, which was the highest value, recorded in 1999 in the Gulf of Lions. Significant declines of the density indices were found only for the Alborán Sea with $\mathrm{r}=0.84(\mathrm{p}<0.05)$.

The mean lengths estimated for the shelf $(28,335$ individuals) and slope (1,432 individuals) strata are shown in Figure 2. The smallest length $(2 \mathrm{~cm})$ was recorded from the Ligurian-North Central Tyrrhenian Sea shelf in 1994 and the largest $(30 \mathrm{~cm})$ from the Catalan Sea slope in 1996. Juveniles ranging in size from $5-8 \mathrm{~cm}$ occurred in most areas.

In all the geographical sectors, the average lengths observed for the shelf were generally smaller than those on the slope. Most of the estimated values for this stratum were greater in the westward geographical sectors. Considering the whole depth range, the mean lengths from west to east showed a highly significant $(\mathrm{p}<0.05)$ decreasing trend, both on the shelf and slope.

\section{Pagellus bogaraveo}

This species was distributed throughout the sampled bathymetric range with higher relative abundance in the deeper strata (200-800 m), except in 
TABLE 4. - Pagellus acarne: mean biomass $\left(\mathrm{kg} / \mathrm{km}^{2}\right)$ estimated from the MEDITS trawl surveys by depth stratum, geographical sector and year (1994-1999). Strata that were not sampled are indicated by '*'. Values higher than $10 \mathrm{~kg} / \mathrm{km}^{2}$ are presented in bold.

\begin{tabular}{|c|c|c|c|c|c|c|c|c|c|c|c|c|c|c|c|c|}
\hline \multirow{2}{*}{$\begin{array}{l}\text { Sector code } \\
111 \mathrm{a}\end{array}$} & \multirow{2}{*}{$\begin{array}{l}\text { Sector } \\
\text { Alborán Sea }\end{array}$} & \multicolumn{5}{|c|}{$\begin{array}{c}1994 \\
\text { Depth (m) }\end{array}$} & \multicolumn{5}{|c|}{$\begin{array}{c}1995 \\
\text { Depth (m) }\end{array}$} & \multicolumn{5}{|c|}{ 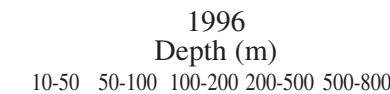 } \\
\hline & & $\mathbf{5 8 3 . 0}$ & 561.9 & 66.5 & 0.5 & $\frac{500-800}{0}$ & $\begin{array}{r}10-50 \\
\mathbf{1 3 5 . 5}\end{array}$ & 281.0 & 2.8 & 0.4 & 0.1 & 199.9 & 131.9 & 118.5 & 3.1 & 0.2 \\
\hline $112 \mathrm{a}$ & Alicante & 3.0 & 0.4 & 0 & 0 & 0 & 23.1 & 20.5 & 0 & 0.1 & 0 & 1.4 & 92.1 & 0 & 1.2 & 0 \\
\hline $113 \mathrm{a}$ & Catalan Sea & 0.1 & 28.1 & 3.5 & 0.9 & 0 & 13.4 & 9.1 & 2.2 & 0.2 & 0 & 1.6 & 18.3 & 20.8 & 3.9 & 0 \\
\hline $114 \mathrm{a}$ & W Morocco & $*$ & $*$ & $*$ & $*$ & $*$ & * & * & * & * & $*$ & * & * & * & * & * \\
\hline $114 \mathrm{~b}$ & E Morocco & $*$ & $*$ & $*$ & $*$ & $*$ & $*$ & * & $*$ & $*$ & $*$ & $*$ & * & $*$ & $*$ & $*$ \\
\hline $121 \mathrm{a}$ & W Gulf of Lions & 8.1 & 26.5 & 3.2 & 0.8 & 0 & 8.3 & 37.1 & 23.8 & 0 & 0 & 9.4 & 9.9 & 5.9 & 0 & 0 \\
\hline $121 \mathrm{~b}$ & E Gulf of Lions & 0.6 & 0.8 & 8.9 & 11.9 & 0 & 0 & 1.9 & 12.2 & 19.8 & 0 & 0 & 0.6 & 13.6 & 29.5 & 0 \\
\hline $131 \mathrm{a}$ & NE Corsica & $*$ & 0 & 0 & 8.6 & 0 & $*$ & 0 & 0.5 & 6.2 & 0 & $*$ & 0.3 & 0 & 0.3 & 0 \\
\hline $131 \mathrm{~b}$ & SE Corsica & $*$ & 0 & 6.1 & 0 & 0 & * & 1.1 & 198.1 & 0 & 0 & $*$ & 67.0 & 0 & 1.1 & 0 \\
\hline $132 \mathrm{a}$ & N Ligurian Sea & 9.5 & 0 & 0 & 0 & 0 & 0 & 0 & 0.5 & 3.5 & 0.2 & 0 & 10.1 & 0 & 5.4 & 0 \\
\hline $132 \mathrm{~b}$ & E Ligurian Sea & 0.8 & 2.2 & 0.8 & 0.2 & 0 & 4.1 & 0 & 0.4 & 0.1 & 0 & 0 & 2.7 & 0.5 & 0.1 & 0 \\
\hline $132 \mathrm{c}$ & N Tyrrhenian & 0 & 0.2 & 5.1 & 0.1 & 0 & 1.2 & 0 & 1.1 & 0 & 0 & 0 & 0 & 1.6 & 0.1 & 0 \\
\hline $132 \mathrm{~d}$ & C Tyrrhenian & 0.9 & 0 & 0.6 & 0 & 0 & 2.6 & 0.9 & 0 & 0.6 & 0 & 0.5 & 0 & 0.5 & 0 & 0 \\
\hline $133 \mathrm{a}$ & SE Sardinia & 2.3 & 0 & 6.6 & 3.3 & 0 & 2.0 & 1.5 & 0 & 1.5 & 0 & 1.6 & 0 & 0 & 0.8 & 0 \\
\hline $133 \mathrm{~b}$ & NE Sardinia & 0 & 0 & 0 & 0 & 0 & 0 & 0 & 0 & 2.3 & 0 & 1.3 & 0 & 0 & 0 & 0 \\
\hline $133 \mathrm{c}$ & N Sardinia & 0 & 1.1 & 0 & 31.3 & 0 & 0.6 & 0 & 0 & 56.3 & 0 & 0 & 36.4 & 0 & 0 & 0 \\
\hline $133 \mathrm{~d}$ & NW Sardinia & 0 & 17.8 & 0 & 0 & 0 & * & 0 & 0 & 0 & 0 & 0 & 0 & 0 & 0 & 0 \\
\hline $133 \mathrm{e}$ & W Sardinia & 17.6 & * & 0.9 & 0 & 0 & 0 & 0 & 0 & 0 & 0 & 11.6 & 0.6 & 0 & 0 & 0 \\
\hline $133 \mathrm{f}$ & SW Sardinia & 0 & 1.0 & 0 & 0 & 0 & 16.0 & 0 & 0 & 0 & 0 & 0 & 0 & 1.0 & 2.4 & 0 \\
\hline $133 \mathrm{~g}$ & S Sardinia & 0 & 0 & 0 & 3.0 & 0 & 6.7 & * & 0 & 0 & 0 & 0.2 & 0 & 0 & 0 & 0 \\
\hline $134 \mathrm{a}$ & SE Tyrrhenian & 3.8 & 0.3 & 0.2 & 4.8 & 0 & 8.3 & 0 & 1.1 & 0.6 & 0 & 7.9 & 0 & 0.6 & 0.5 & 0 \\
\hline $134 \mathrm{~b}$ & SW Tyrrhenian & 179.3 & 16.1 & 0 & 0 & 0 & 16.5 & 7.1 & 2.6 & 1.3 & 0 & 0 & 0.3 & 1.1 & 0 & 0 \\
\hline $134 \mathrm{c}$ & Sicilian Chan. & 0.4 & 1.2 & 0 & 0.1 & 0 & 0 & 0.9 & 0.1 & 0.1 & 0 & 0 & 2.2 & 0.0 & 1.2 & 0 \\
\hline $211 \mathrm{a}$ & N Adriatic Sea & 0 & 0.2 & * & $*$ & $*$ & 0 & 0 & * & * & $*$ & 0.1 & 0 & * & * & * \\
\hline $211 \mathrm{~b}$ & Central Adriatic & 0 & 0.2 & 0 & 0 & 0 & 0 & 0 & 0 & 0 & 0 & 0.4 & 0.5 & 0.1 & 0.4 & 0 \\
\hline $211 \mathrm{c}$ & N Adriatic-Slov & * & $*$ & $*$ & * & * & 0 & * & * & * & $*$ & 0 & * & * & * & * \\
\hline $211 d$ & NE Adri Croatia & $*$ & * & $*$ & $*$ & $*$ & * & * & * & * & $*$ & 0.3 & 1.2 & 0 & 0.1 & $*$ \\
\hline $221 \mathrm{a}$ & E Sicily & 2.0 & 3.3 & 0 & 0 & 0 & 1.2 & 4.0 & 0.7 & 0 & 0 & 5.5 & 0 & 0 & 0 & 0 \\
\hline $221 b$ & NW Ionian Sea & 0 & 0 & 0 & 0 & 0 & 0 & 1.1 & 0 & 0 & 0 & 0.8 & 0 & 0 & 0.4 & 0 \\
\hline $221 \mathrm{c}$ & N Ionian Sea & 0.4 & 0.5 & 0 & 0 & 0 & 0 & 0.4 & 0.6 & 0 & 0 & 0 & 0 & 0 & 0 & 0 \\
\hline $221 d$ & N Ionian Sea & $*$ & 0 & 0 & 0 & 0 & 0 & 0 & 0 & 0.1 & 0 & 0 & 1.1 & 0 & 0 & 0 \\
\hline $221 \mathrm{e}$ & SW Adriatic & 0 & 6.7 & 0 & 0 & 0 & * & 0 & 0.4 & 0 & 0 & $*$ & 0 & 0 & 0 & 0 \\
\hline $221 \mathrm{f}$ & SW Adriatic & 0 & 0 & 0 & 0 & 0 & 0 & 0 & 0 & 0 & 0 & 0 & 0 & 0 & 0 & 0 \\
\hline $221 \mathrm{~g}$ & SW Adriatic & 0.5 & 0 & 0 & $*$ & 0 & 0 & 0 & 0 & * & 0 & 0 & 0 & 0 & * & 0 \\
\hline $221 \mathrm{~h}$ & SW Adriatic & 0.1 & 0 & 0 & 4.5 & 0 & 0 & 0.1 & 0 & 22.4 & 0 & 0.3 & 0 & 0 & 0 & 0 \\
\hline $221 \mathrm{i}$ & SE Adriatic & $*$ & $*$ & $*$ & $*$ & $*$ & $*$ & * & * & $*$ & $*$ & 0.4 & 0 & 0 & 0 & 0 \\
\hline $222 \mathrm{a}$ & E Ionian Sea & 0.4 & 38.9 & 0 & 0 & 0 & 0.4 & 0.6 & 0 & 0 & 0 & 0 & 4.4 & 0.8 & 0 & 0 \\
\hline $223 \mathrm{a}$ & Argosaronikos & 0 & 4.0 & 0 & 0 & 0 & 12.7 & 0 & 0 & 0 & 0 & 0 & 1.1 & 0 & 0 & 0 \\
\hline $224 a$ & N Aegean Sea & 0 & 0 & 0 & 0 & 0 & 0 & 5.1 & 0.3 & 1.0 & 0 & 0.4 & 0.6 & 0.6 & 0.6 & 0 \\
\hline $225 \mathrm{a}$ & S Aegean Sea & 49.6 & 55.1 & 0.3 & 0.3 & 0 & 47.8 & 1.2 & 1.6 & 0.0 & 0 & 0 & 31.1 & 29.2 & 10.9 & 0 \\
\hline Sector code & Sector & $10-50$ & D & 1997 & & $500-800$ & $10-50$ & $50-100$ & $\begin{array}{l}1998 \\
\text { Depth }\end{array}$ & (m) & & $10-50$ & & $\begin{array}{r}1999 \\
\text { Depth }(1\end{array}$ & & \\
\hline $111 \mathrm{a}$ & Alborán Sea & 57.4 & 68.3 & 164.5 & 2.2 & 0.5 & 945.2 & 86.3 & 137.2 & 1.7 & 0.6 & 32.1 & 11.3 & 85.4 & 1.8 & 0.4 \\
\hline $112 \mathrm{a}$ & Alicante & 0.6 & 5.5 & 0.8 & 0 & 0 & 2.1 & 28.2 & 0 & 0.4 & 0.1 & 42.2 & 47.2 & 0.3 & 0.1 & 0 \\
\hline $113 a$ & Catalan Sea & 3.9 & 43.5 & 2.2 & 0 & 0 & 24.6 & 23.7 & 0 & $*$ & 0 & 16.1 & 6.8 & 1.1 & 0 & 0 \\
\hline $114 a$ & W Morocco & * & * & $*$ & $*$ & $*$ & $*$ & * & * & * & $*$ & * & 107.7 & 79.7 & 2.3 & 0 \\
\hline $114 \mathrm{~b}$ & E Morocco & $*$ & * & $*$ & $*$ & $*$ & $*$ & * & * & $*$ & $*$ & 600.9 & 0 & 0 & 3.7 & 0 \\
\hline $121 \mathrm{a}$ & W Gulf of Lions & 14.2 & 38.7 & 6.5 & 0 & 0 & 38.8 & 17.4 & 0 & 0 & 0 & 49.9 & 20.6 & 7.8 & 0 & 0 \\
\hline $121 \mathrm{~b}$ & E Gulf of Lions & 0.5 & 2.0 & 7.3 & 25.2 & 0 & 0.4 & 2.2 & 7.8 & 0 & 0 & 1.9 & 0.2 & 12.8 & 36.1 & * \\
\hline $131 \mathrm{a}$ & NE Corsica & $*$ & 0 & $*$ & 0 & 0 & $*$ & 0 & 0 & 0.1 & 0 & $*$ & 0 & 0 & 4.2 & 0 \\
\hline $131 \mathrm{~b}$ & SE Corsica & $*$ & 0 & 0 & 0 & $*$ & $*$ & 10.9 & 0 & 1.9 & 0 & $*$ & 0 & 16.1 & 0 & 0 \\
\hline $132 \mathrm{a}$ & N Ligurian Sea & 31.5 & 0.3 & 0 & 0 & 0 & 0 & 1.3 & 0 & 4.8 & 0 & 0 & 0.4 & 0 & 0 & 0 \\
\hline $132 \mathrm{~b}$ & E Ligurian Sea & 11.5 & 0.5 & 0.8 & 0.2 & 0 & 0.8 & 1.1 & 0 & 0.1 & 0 & 3.8 & 2.0 & 0.3 & 1.9 & 0 \\
\hline $132 \mathrm{c}$ & N Tyrrhenian & 7.5 & 0.2 & 1.3 & 0.2 & 0 & 4.2 & 0 & 1.7 & 0 & 0 & 2.7 & 1.8 & 2.8 & 0 & 0 \\
\hline $132 \mathrm{~d}$ & C Tyrrhenian & 2.7 & 0 & 0 & 0 & 0 & 28.0 & 0.4 & 5.4 & 0.1 & 0 & 6.7 & 0.3 & 0.9 & 0.1 & 0 \\
\hline $133 \mathrm{a}$ & SE Sardinia & 0 & 0 & 0 & 0 & 0 & 1.0 & 1.2 & 0 & 0 & 0 & 19.5 & 0 & 0 & 0.7 & 0 \\
\hline $133 \mathrm{~b}$ & NE Sardinia & 28.0 & 1.9 & 0 & 0 & 0 & 12.4 & 0 & 0 & 0 & 0 & 0 & 0.8 & 10.5 & 0 & 0 \\
\hline $133 \mathrm{c}$ & N Sardinia & 0 & 1.4 & 0 & 0 & 0 & 0.9 & 0 & 0 & 0 & 0 & 14.2 & 0.9 & 1.0 & 76.0 & 0 \\
\hline $133 \mathrm{~d}$ & NW Sardinia & 0 & 0.6 & 0 & 0 & 0 & 0 & 5.4 & 6.5 & 0 & 0 & 0 & 0 & 0 & 0 & 0 \\
\hline $133 \mathrm{e}$ & W Sardinia & 0.7 & 0 & 0 & 0 & 0 & 4.9 & 0.5 & 0 & 0 & 0 & 7.2 & 23.9 & 0 & 0 & 0 \\
\hline $133 \mathrm{f}$ & SW Sardinia & 1.3 & 3.5 & 0.1 & 0 & 0 & 10.1 & 0 & 0.5 & 0 & 0 & 4.3 & 0 & 1.2 & 0.7 & 0 \\
\hline $133 \mathrm{~g}$ & S Sardinia & 0 & 0 & 0 & 0.3 & 0 & 0 & 0 & 0 & 0 & 0 & 0.4 & 0 & 0.2 & 0 & 0 \\
\hline $134 \mathrm{a}$ & SE Tyrrhenian & 10.31 & 0 & 2.3 & 0.5 & 0 & 4.4 & 0.2 & 0.5 & 0.1 & 0 & 18.1 & 4.0 & 0.3 & 0.1 & 0 \\
\hline $134 \mathrm{~b}$ & SW Tyrrhenian & 88.3 & 12.3 & 0.5 & 21.7 & 0 & 403.9 & 27.2 & 6.4 & 0.2 & 0 & 0 & 4.6 & 6.5 & 0 & 0 \\
\hline
\end{tabular}


TABLE 4 (Cont.). - Pagellus acarne: mean biomass $\left(\mathrm{kg} / \mathrm{km}^{2}\right)$ estimated from the MEDITS trawl surveys by depth stratum, geographical sector and year (1994-1999). Strata that were not sampled are indicated by '*'. Values higher than $10 \mathrm{~kg} / \mathrm{km}^{2}$ are presented in bold.

\begin{tabular}{|c|c|c|c|c|c|c|c|c|c|c|c|c|c|c|c|c|}
\hline \multirow{2}{*}{$\frac{\text { Sector code }}{134 \mathrm{c}}$} & \multirow{2}{*}{$\begin{array}{l}\text { Sector } \\
\text { Sicilian Chan. }\end{array}$} & \multicolumn{5}{|c|}{ 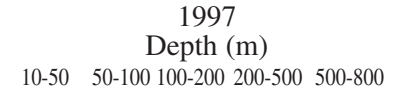 } & \multicolumn{5}{|c|}{$\begin{array}{c}1998 \\
\text { Depth (m) }\end{array}$} & \multicolumn{4}{|c|}{$\begin{array}{c}1999 \\
\text { Depth (m) }\end{array}$} & \\
\hline & & 0.6 & 2.6 & 0 & 0 & 0 & 0 & 2.7 & 0.1 & 0 & 0 & 0 & 7.3 & 0 & 0 & 0 \\
\hline $211 \mathrm{a}$ & N Adriatic Sea & 0 & 0.1 & * & * & * & 0 & 1.4 & * & * & $*$ & 0.3 & 0 & $*$ & * & $*$ \\
\hline $211 b$ & Central Adriatic & 0 & 0.8 & 0.1 & 0.3 & 0 & 0 & 0.1 & 0.1 & 0 & $*$ & 1.9 & 0 & 0 & 0.1 & $*$ \\
\hline $211 \mathrm{c}$ & N Adriatic-Slov & 0 & $*$ & * & * & * & 0 & * & $*$ & $*$ & $*$ & 0 & * & $*$ & * & * \\
\hline $211 d$ & NE Adri Croatia & 0 & 0.5 & 0 & 0 & * & 0.2 & 0.4 & 0.2 & 0 & * & 0 & $*$ & $*$ & $*$ & $*$ \\
\hline $221 \mathrm{a}$ & E Sicily & 59.34 & 0.7 & 3.1 & 0 & 0 & 17.2 & 7.9 & 4.7 & 5.0 & 0 & 0.5 & 0.1 & 0.8 & 0 & 0 \\
\hline $221 b$ & NW Ionian Sea & 0 & 0 & 0 & 0 & 0 & 0.5 & 0 & 1.5 & 0 & 0 & 0.5 & 0 & 0.7 & 0 & 0 \\
\hline $221 \mathrm{c}$ & N Ionian Sea & 0.4 & 0.8 & 0.6 & 0 & 0 & 0.7 & 0.6 & 1.0 & 0 & 0 & 0 & 0 & 0.8 & 0 & 0 \\
\hline $221 d$ & N Ionian Sea & 0 & 0 & 0.7 & 0 & 0 & 0 & 0 & 0 & 0 & 0.1 & 0 & 0 & 1.0 & 0 & 0 \\
\hline $221 \mathrm{e}$ & SW Adriatic & * & 0.3 & 0.2 & 0 & 0 & * & 0 & 0 & 0 & 0 & $*$ & 0 & 0 & 0 & 0 \\
\hline $221 \mathrm{f}$ & SW Adriatic & 0 & 0 & 0 & 0 & 0 & 0 & 0 & 0 & 0 & 0 & 2.5 & 0 & 0 & 0 & 0 \\
\hline $221 \mathrm{~g}$ & SW Adriatic & 0 & 0 & 0 & $*$ & 0 & 0 & 0 & 0 & * & 0 & 9.1 & 0 & 0 & * & 0 \\
\hline $221 \mathrm{~h}$ & SW Adriatic & 0 & 0.3 & 0 & 1.2 & 0 & 0.1 & 0 & 0 & 0.4 & 0 & 0 & 0.4 & 0 & 0.2 & 0 \\
\hline $221 \mathrm{i}$ & SE Adriatic & 0 & 0.3 & 0 & 0 & 0 & 0 & 0.5 & 0.3 & 0 & 0 & 0.5 & 0 & 0 & 0.2 & 0 \\
\hline $222 \mathrm{a}$ & E Ionian Sea & 0 & 0 & 0 & 0 & 0 & 19.8 & 1.3 & 0.4 & 0 & 0 & 82.3 & 3.6 & 2.3 & 0.3 & 0 \\
\hline $223 a$ & Argosaronikos & 37.66 & 3.4 & 0 & 0 & 0 & 12.2 & 0 & 0.1 & 0.3 & 0 & 193.8 & 0 & 0 & 1.5 & 0 \\
\hline $224 \mathrm{a}$ & N Aegean Sea & 6.2 & 0.1 & 0.1 & 0 & 0 & 6.0 & 0 & 0.1 & 0 & 0 & 1.4 & 0.4 & 0 & 0 & 0 \\
\hline $225 \mathrm{a}$ & S Aegean Sea & 0 & 2.6 & 2.1 & 0.1 & 0 & 286.4 & 62.9 & 0.4 & 0.8 & 0 & 17.6 & 15.4 & 0.1 & 3.9 & 0 \\
\hline
\end{tabular}

TABLE 5. - Pagellus acarne: mean density (in number of individuals $/ \mathrm{km}^{2}$ ) estimated from the MEDITS trawl surveys by depth stratum, geographical sector and year (1994-1999). Strata that were not sampled are indicated by '*'. Values higher than 200 individuals $/ \mathrm{km}^{2}$ are presented in bold.

\begin{tabular}{|c|c|c|c|c|c|c|c|c|c|c|c|c|c|c|c|c|}
\hline \multirow{2}{*}{$\begin{array}{l}\text { Sector code } \\
111 \mathrm{a}\end{array}$} & \multirow{2}{*}{$\begin{array}{l}\text { Sector } \\
\text { Alborán Sea }\end{array}$} & \multicolumn{5}{|c|}{$\begin{array}{c}1994 \\
\text { Depth (m) }\end{array}$} & \multicolumn{5}{|c|}{$\begin{array}{c}1995 \\
\text { Depth (m) }\end{array}$} & \multicolumn{5}{|c|}{$\begin{array}{c}1996 \\
\text { Depth (m) }\end{array}$} \\
\hline & & 8427 & 2991 & 423 & 5 & 0 & 9908 & 4386 & 38 & 3 & 1 & 2528 & 1958 & 654 & 24 & 3 \\
\hline $112 \mathrm{a}$ & Alicante & 192 & 4 & 0 & 0 & 0 & 1693 & 228 & 0 & 1 & 0 & 33 & 603 & 0 & 8 & 0 \\
\hline $113 a$ & Catalan Sea & 22 & 252 & 32 & 5 & 0 & 172 & 76 & 14 & 2 & 0 & 21 & 125 & 112 & 11 & 0 \\
\hline $114 \mathrm{a}$ & W Morocco & * & $*$ & * & * & * & * & * & * & * & $*$ & * & $*$ & * & $*$ & * \\
\hline $114 b$ & E Morocco & $*$ & $*$ & $*$ & $*$ & $*$ & $*$ & $*$ & $*$ & $*$ & $*$ & $*$ & $*$ & $*$ & $*$ & * \\
\hline $121 \mathrm{a}$ & W Gulf of Lions & 80 & 91 & 21 & 7 & 0 & 60 & 145 & 104 & 0 & 0 & 110 & 48 & 9 & 0 & 0 \\
\hline $121 \mathrm{~b}$ & E Gulf of Lions & 6 & 4 & 30 & 36 & 0 & 0 & 8 & 39 & 61 & 0 & 0 & 2 & 41 & 73 & 0 \\
\hline $131 \mathrm{a}$ & NE Corsica & * & 0 & 0 & 52 & 0 & * & 0 & 10 & 40 & 0 & $*$ & 7 & 0 & 2 & 0 \\
\hline $131 \mathrm{~b}$ & SE Corsica & * & 0 & 122 & 0 & 0 & * & 21 & 2218 & 0 & 0 & $*$ & 1680 & 0 & 6 & 0 \\
\hline $132 \mathrm{a}$ & N Ligurian Sea & 9154 & 0 & 0 & 0 & 0 & 0 & 0 & 6 & 12 & 1 & 0 & 512 & 0 & 14 & 0 \\
\hline $132 b$ & E Ligurian Sea & 22 & 34 & 11 & 1 & 0 & 103 & 0 & 3 & 1 & 0 & 3 & 26 & 8 & 1 & 0 \\
\hline $132 \mathrm{c}$ & $\mathrm{N}$ Tyrrhenian & 0 & 3 & 28 & 1 & 0 & 112 & 0 & 4 & 0 & 0 & 0 & 0 & 8 & 1 & 0 \\
\hline $132 d$ & C Tyrrhenian & 11 & 0 & 6 & 2 & 0 & 42 & 10 & 0 & 3 & 0 & 11 & 0 & 5 & 0 & 0 \\
\hline $133 a$ & SE Sardinia & 65 & 0 & 202 & 16 & 0 & 104 & 23 & 0 & 11 & 0 & 96 & 0 & 0 & 4 & 0 \\
\hline $133 b$ & NE Sardinia & 0 & 0 & 0 & 0 & 0 & 0 & 0 & 0 & 7 & 0 & 26 & 0 & 0 & 0 & 0 \\
\hline $133 \mathrm{c}$ & N Sardinia & 0 & 8 & 0 & 259 & 0 & 8 & 0 & 0 & 408 & 0 & 0 & 485 & 0 & 0 & 0 \\
\hline $133 d$ & NW Sardinia & 0 & 227 & 0 & 0 & 0 & * & 0 & 0 & 0 & 0 & 0 & 0 & 0 & 0 & 0 \\
\hline $133 \mathrm{e}$ & W Sardinia & 392 & * & 4 & 0 & 0 & 0 & 0 & 0 & 0 & 0 & 308 & 13 & 0 & 0 & 0 \\
\hline $133 \mathrm{f}$ & SW Sardinia & 0 & 12 & 0 & 0 & 0 & 324 & 0 & 0 & 0 & 0 & 0 & 0 & 12 & 16 & 0 \\
\hline $133 \mathrm{~g}$ & S Sardinia & 0 & 0 & 0 & 9 & 0 & 598 & $*$ & 0 & 0 & 0 & 24 & 0 & 0 & 0 & 0 \\
\hline $134 a$ & SE Tyrrhenian & 294 & 4 & 4 & 40 & 0 & 581 & 0 & 17 & 7 & 0 & 511 & 0 & 7 & 3 & 0 \\
\hline $134 b$ & SW Tyrrhenian & 11633 & 272 & 0 & 0 & 0 & 512 & 153 & 44 & 8 & 0 & 0 & 6 & 14 & 0 & 0 \\
\hline $134 \mathrm{c}$ & Sicilian Chan. & 6 & 12 & 0 & 1 & 0 & 0 & 13 & 2 & 1 & 0 & 0 & 32 & 0 & 7 & 0 \\
\hline $211 \mathrm{a}$ & N Adriatic Sea & 0 & 2 & * & * & * & 0 & 0 & * & * & $*$ & 1 & 0 & * & * & * \\
\hline $211 b$ & Central Adriatic & 0 & 1 & 0 & 0 & 0 & 0 & 0 & 0 & 0 & 0 & 6 & 3 & 2 & 6 & 0 \\
\hline $211 \mathrm{c}$ & N Adriatic-Slov & * & $*$ & * & $*$ & * & 0 & * & * & * & $*$ & 0 & $*$ & * & $*$ & $*$ \\
\hline $211 d$ & NE Adri Croatia & * & * & * & * & * & * & * & * & * & $*$ & 6 & 30 & 0 & 3 & * \\
\hline $221 \mathrm{a}$ & E Sicily & 402 & 33 & 0 & 0 & 0 & 24 & 55 & 7 & 0 & 0 & 88 & 0 & 0 & 0 & 0 \\
\hline $221 b$ & NW Ionian Sea & 0 & 0 & 0 & 0 & 0 & 0 & 11 & 0 & 0 & 0 & 22 & 0 & 0 & 4 & 0 \\
\hline $221 \mathrm{c}$ & N Ionian Sea & 8 & 8 & 0 & 0 & 0 & 0 & 7 & 12 & 0 & 0 & 0 & 0 & 0 & 0 & 0 \\
\hline $221 d$ & N Ionian Sea & * & 0 & 0 & 0 & 0 & 0 & 0 & 0 & 2 & 0 & 0 & 19 & 0 & 0 & 0 \\
\hline $221 \mathrm{e}$ & SW Adriatic & 0 & 90 & 0 & 0 & 0 & * & 0 & 3 & 0 & 0 & * & 0 & 0 & 0 & 0 \\
\hline $221 \mathrm{f}$ & SW Adriatic & 0 & 0 & 0 & 0 & 0 & 0 & 0 & 0 & 0 & 0 & 0 & 0 & 0 & 0 & 0 \\
\hline $221 \mathrm{~g}$ & SW Adriatic & 37 & 0 & 0 & * & 0 & 0 & 0 & 0 & * & 0 & 0 & 0 & 0 & * & 0 \\
\hline $221 \mathrm{~h}$ & SW Adriatic & 5 & 0 & 0 & 29 & 0 & 3 & 4 & 0 & 85 & 0 & 10 & 0 & 0 & 0 & 0 \\
\hline $221 \mathrm{i}$ & SE Adriatic & * & * & * & $*$ & $*$ & * & $*$ & * & * & $*$ & 7 & 0 & 0 & 0 & 0 \\
\hline $222 \mathrm{a}$ & E Ionian Sea & 17 & 416 & 0 & 0 & 0 & 35 & 6 & 0 & 0 & 0 & 0 & 71 & 8 & 0 & 0 \\
\hline $223 a$ & Argosaronikos & 0 & 21 & 0 & 0 & 0 & 440 & 0 & 0 & 0 & 0 & 0 & 47 & 0 & 0 & 0 \\
\hline $224 a$ & N Aegean Sea & 0 & 0 & 0 & 0 & 0 & 0 & 61 & 7 & 13 & 0 & 7 & 12 & 10 & 12 & 0 \\
\hline $225 \mathrm{a}$ & S Aegean Sea & 2373 & 1226 & 7 & 2 & 0 & 512 & 54 & 25 & 0 & 0 & 0 & 2175 & 524 & 170 & 0 \\
\hline
\end{tabular}


TABle 5 (Cont.). - Pagellus acarne: mean density (in number of individuals $/ \mathrm{km}^{2}$ ) estimated from the MEDITS trawl surveys by depth stratum, geographical sector and year (1994-1999). Strata that were not sampled are indicated by '*'. Values higher than 200 individuals /km² are presented in bold.

\begin{tabular}{|c|c|c|c|c|c|c|c|c|c|c|c|c|c|c|c|c|}
\hline \multirow{2}{*}{$\begin{array}{l}\text { Sector code } \\
111 \mathrm{a}\end{array}$} & \multirow{2}{*}{$\begin{array}{l}\text { Sector } \\
\text { Alborán Sea }\end{array}$} & \multicolumn{5}{|c|}{$\begin{array}{c}1997 \\
\text { Depth (m) }\end{array}$} & \multicolumn{5}{|c|}{$\begin{array}{c}1998 \\
\text { Depth (m) }\end{array}$} & \multicolumn{5}{|c|}{$\begin{array}{c}1999 \\
\text { Depth (m) }\end{array}$} \\
\hline & & $\begin{array}{r}10-50 \\
\mathbf{8 6 3}\end{array}$ & 900 & 703 & 7 & 5 & $\begin{array}{r}10-50 \\
6091\end{array}$ & 1107 & 831 & 17 & 5 & $\begin{array}{r}10-50 \\
\mathbf{4 6 5}\end{array}$ & $\begin{array}{r}50-100 \\
125\end{array}$ & 414 & 7 & 3 \\
\hline $112 \mathrm{a}$ & Alicante & 9 & 57 & 8 & 0 & 0 & 22 & 172 & 0 & 1 & 2 & 636 & 548 & 3 & 1 & 0 \\
\hline $113 a$ & Catalan Sea & 42 & 277 & 17 & 0 & 0 & 239 & 107 & 0 & $*$ & 0 & 190 & 59 & 4 & 0 & 0 \\
\hline $114 \mathrm{a}$ & W Morocco & $*$ & $*$ & $*$ & $*$ & $*$ & $*$ & $*$ & $*$ & $*$ & $*$ & $*$ & 63 & 500 & 10 & 0 \\
\hline $114 b$ & E Morocco & $*$ & $*$ & $*$ & $*$ & $*$ & $*$ & $*$ & $*$ & $*$ & $*$ & 31160 & 0 & 0 & 35 & 0 \\
\hline $121 \mathrm{a}$ & W Gulf of Lions & 108 & 163 & 27 & 0 & 0 & 383 & 79 & 0 & 0 & 0 & 389 & 78 & 27 & 0 & 0 \\
\hline $121 b$ & E Gulf of Lions & 3 & 8 & 26 & 72 & 0 & 4 & 12 & 26 & 0 & 0 & 21 & 2 & 67 & 114 & $*$ \\
\hline $131 \mathrm{a}$ & NE Corsica & $*$ & 0 & * & 0 & 0 & $*$ & 0 & 0 & 1 & 0 & $*$ & 0 & 0 & 28 & 0 \\
\hline $131 b$ & SE Corsica & $*$ & 0 & 0 & 0 & $*$ & $*$ & 384 & 0 & 13 & 0 & $*$ & 0 & 264 & 0 & 0 \\
\hline $132 \mathrm{a}$ & N Ligurian Sea & 1487 & 8 & 0 & 0 & 0 & 0 & 42 & 0 & 13 & 0 & 0 & 7 & 0 & 0 & 0 \\
\hline $132 b$ & E Ligurian Sea & 679 & 6 & 9 & 2 & 0 & 33 & 16 & 0 & 1 & 0 & 58 & 28 & 4 & 16 & 0 \\
\hline $132 \mathrm{c}$ & $\mathrm{N}$ Tyrrhenian & 138 & 3 & 8 & 2 & 0 & 291 & 0 & 12 & 0 & 0 & 87 & 27 & 27 & 0 & 0 \\
\hline $132 d$ & C Tyrrhenian & 81 & 0 & 0 & 0 & 0 & 555 & 4 & 48 & 1 & 0 & 175 & 3 & 9 & 2 & 0 \\
\hline $133 a$ & SE Sardinia & 0 & 0 & 0 & 0 & 0 & 123 & 23 & 0 & 0 & 0 & 2018 & 0 & 0 & 3 & 0 \\
\hline $133 b$ & NE Sardinia & 594 & 34 & 0 & 0 & 0 & 273 & 0 & 0 & 0 & 0 & 0 & 11 & 84 & 0 & 0 \\
\hline $133 \mathrm{c}$ & N Sardinia & 0 & 24 & 0 & 0 & 0 & 18 & 0 & 0 & 0 & 0 & 225 & 7 & 13 & 630 & 0 \\
\hline $133 d$ & NW Sardinia & 0 & 8 & 0 & 0 & 0 & 0 & 76 & 31 & 0 & 0 & 0 & 0 & 0 & 0 & 0 \\
\hline $133 \mathrm{e}$ & W Sardinia & 14 & 0 & 0 & 0 & 0 & 221 & 14 & 0 & 0 & 0 & 151 & 468 & 0 & 0 & 0 \\
\hline $133 \mathrm{f}$ & SW Sardinia & 26 & 82 & 2 & 0 & 0 & 269 & 0 & 6 & 0 & 0 & 97 & 0 & 16 & 5 & 0 \\
\hline $133 \mathrm{~g}$ & S Sardinia & 0 & 0 & 0 & 3 & 0 & 0 & 0 & 0 & 0 & 0 & 60 & 0 & 4 & 0 & 0 \\
\hline $134 \mathrm{a}$ & SE Tyrrhenian & 370 & 0 & 36 & 7 & 0 & 442 & 4 & 9 & 1 & 0 & 466 & 83 & 5 & 1 & 0 \\
\hline $134 b$ & SW Tyrrhenian & 1076 & 180 & 7 & 184 & 0 & 12029 & 275 & 132 & 1 & 0 & 0 & 126 & 92 & 0 & 0 \\
\hline $134 \mathrm{c}$ & Sicilian Chan. & 6 & 43 & 0 & 0 & 1 & 0 & 36 & 2 & 0 & 0 & 0 & 102 & 0 & 1 & 0 \\
\hline $211 \mathrm{a}$ & N Adriatic Sea & 0 & 2 & $*$ & $*$ & $*$ & 0 & 23 & $*$ & $*$ & $*$ & 13 & 0 & $*$ & $*$ & $*$ \\
\hline $211 b$ & Central Adriatic & 0 & 13 & 1 & 4 & 0 & 0 & 2 & 1 & 0 & $*$ & 94 & 0 & 0 & 2 & $*$ \\
\hline $211 \mathrm{c}$ & N Adriatic-Slov & 0 & $*$ & $*$ & $*$ & $*$ & 0 & $*$ & $*$ & $*$ & $*$ & 0 & $*$ & $*$ & $*$ & * \\
\hline $211 d$ & NE Adri Croatia & 0 & 10 & 1 & 0 & $*$ & 5 & 7 & 4 & 0 & $*$ & 0 & $*$ & $*$ & $*$ & $*$ \\
\hline $221 \mathrm{a}$ & E Sicily & 6211 & 42 & 63 & 0 & 0 & 2556 & 157 & 84 & 43 & 0 & 8 & 11 & 15 & 0 & 0 \\
\hline $221 b$ & NW Ionian Sea & 0 & 0 & 0 & 0 & 0 & 11 & 0 & 32 & 0 & 0 & 11 & 0 & 11 & 0 & 0 \\
\hline $221 \mathrm{c}$ & N Ionian Sea & 7 & 15 & 11 & 0 & 0 & 15 & 15 & 21 & 0 & 0 & 0 & 0 & 11 & 0 & 0 \\
\hline $221 d$ & N Ionian Sea & 0 & 0 & 7 & 0 & 0 & 0 & 0 & 0 & 0 & 1 & 0 & 0 & 23 & 0 & 0 \\
\hline $221 \mathrm{e}$ & SW Adriatic & $*$ & 7 & 3 & 0 & 0 & $*$ & 0 & 0 & 0 & 0 & $*$ & 0 & 0 & 0 & 0 \\
\hline $221 \mathrm{f}$ & SW Adriatic & 0 & 0 & 0 & 0 & 0 & 0 & 0 & 0 & 0 & 0 & 270 & 0 & 0 & 0 & 0 \\
\hline $221 \mathrm{~g}$ & SW Adriatic & 0 & 0 & 0 & $*$ & 0 & 0 & 0 & 0 & $*$ & 0 & 520 & 0 & 0 & $*$ & 0 \\
\hline $221 \mathrm{~h}$ & SW Adriatic & 0 & 4 & 0 & 15 & 0 & 3 & 0 & 0 & 6 & 0 & 3 & 8 & 0 & 3 & 0 \\
\hline $221 \mathrm{i}$ & SE Adriatic & 0 & 2 & 0 & 0 & 0 & 0 & 9 & 4 & 0 & 0 & 15 & 2 & 0 & 1 & 0 \\
\hline $222 \mathrm{a}$ & E Ionian Sea & 0 & 0 & 0 & 0 & 0 & 1331 & 32 & 16 & 0 & 0 & 7594 & 104 & 169 & 5 & 0 \\
\hline $223 a$ & Argosaronikos & 910 & 34 & 0 & 0 & 0 & 714 & 0 & 3 & 6 & 0 & 5116 & 0 & 0 & 39 & 0 \\
\hline $224 a$ & N Aegean Sea & 146 & 2 & 2 & 0 & 0 & 148 & 0 & 2 & 0 & 0 & 51 & 9 & 0 & 0 & 0 \\
\hline $225 a$ & S Aegean Sea & 0 & 45 & 44 & 3 & 0 & 20536 & 2966 & 10 & 18 & 0 & 1542 & 1029 & 3 & 56 & 0 \\
\hline
\end{tabular}

1994 in the Alborán Sea, where a biomass of 135.5 $\mathrm{kg} \cdot \mathrm{km}^{-2}$ (density: 4,545 individuals $\cdot \mathrm{km}^{-2}$ ) was recorded (Tables 6 and 7). Considering the whole shelf, a great difference in biomass (maximum 33.9 $\left.\mathrm{kg} \cdot \mathrm{km}^{-2} ; \mathrm{CV} \%=75.7\right)$ and density (maximum 614 individuals $\left.\cdot \mathrm{km}^{-2} ; \mathrm{CV} \%=69.2\right)$ indices between the Alborán Sea and all the other regions was recorded. Captures from the slope were generally greater than those from the shelf, particularly in Corsica waters and in the Alborán Sea, where values as high as $181.4(\mathrm{CV} \%=51.0)$ in 1998 and $146.0 \mathrm{~kg} \cdot \mathrm{km}^{-2}$ $(\mathrm{CV}=106.3)$ in 1996 were recorded. Remarkable biomass indices were also obtained on the slope in the Gulf of Lions, especially in 1998 and 1999 , when values of $33.7(\mathrm{CV} \%=128.4)$ and $28.6 \mathrm{~kg} \cdot \mathrm{km}^{-}$ ${ }^{2}(\mathrm{CV} \%=84.9)$ were recorded. Concerning the density indices, the highest values were observed on the slope of the Alborán Sea (205 individuals $\cdot \mathrm{km}^{-2}$, CV\%=98.5) in 1996, in the North Aegean Sea (182 individuals $\cdot \mathrm{km}^{-2}, \mathrm{CV} \%=38.0$ ) in 1999 , and in the South Aegean Sea (193 individuals $\mathrm{km}^{-2}$, $\mathrm{CV} \%=38.0)$ in 1998.

Mean lengths (Fig. 3) correspond to 2,937 individuals from the shelf and to 4,017 individuals from the slope. The smallest fish $(3 \mathrm{~cm})$ were observed in the Ligurian-North Central Tyrrhenian Sea and the South Tyrrhenian Sea shelves, in 1994 and 1996, respectively. The largest length $(48 \mathrm{~cm})$ was recorded on the slope in the Gulf of Lions in 1996 and 1998. Generally, the mean lengths of fish from the shelf were lower than those from the slope. The mean lengths from west to east showed a highly significant $(\mathrm{p}<0.05)$ decreasing trend both on the shelf and slope. 

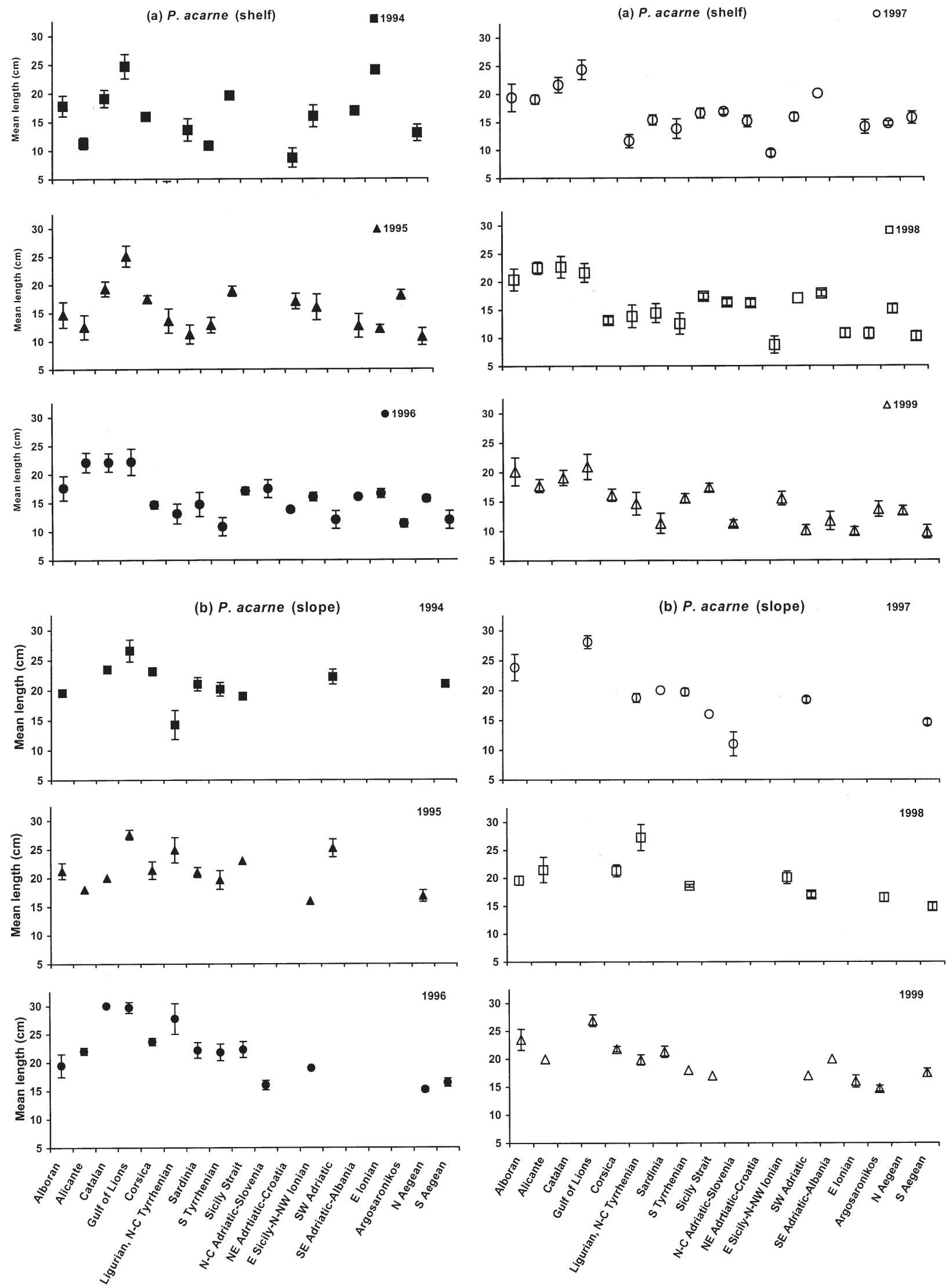

FIG. 2. - Mean total length ( \pm S.D.) of Pagellus acarne on the shelf (a) and slope (b) by geographical region and year. 
TABLE 6. - Pagellus bogaraveo: mean biomass $\left(\mathrm{kg} / \mathrm{km}^{2}\right)$ estimated from the MEDITS trawl surveys by depth stratum, geographical sector and year (1994-1999). Strata that were not sampled are indicated by ' $*$ '. Values higher than $10 \mathrm{~kg} / \mathrm{km}^{2}$ are presented in bold.

\begin{tabular}{|c|c|c|c|c|c|c|c|c|c|c|c|c|c|c|c|c|}
\hline \multirow{3}{*}{$\begin{array}{l}\text { Sector code } \\
111 \mathrm{a}\end{array}$} & \multirow{3}{*}{$\begin{array}{l}\text { Sector } \\
\text { Alborán Sea }\end{array}$} & \multicolumn{5}{|c|}{$\begin{array}{c}1994 \\
\text { Depth (m) }\end{array}$} & \multicolumn{5}{|c|}{$\begin{array}{c}1995 \\
\text { Depth (m) }\end{array}$} & \multicolumn{5}{|c|}{$\begin{array}{c}1996 \\
\text { Depth (m) }\end{array}$} \\
\hline & & $10-50$ & \multicolumn{3}{|c|}{$50-100100-200200-500$} & \multirow{2}{*}{$\begin{array}{r}500-800 \\
\mathbf{3 9 . 0}\end{array}$} & \multirow{2}{*}{$\begin{array}{r}10-50 \\
0\end{array}$} & $50-100$ & 00-200 2 & $200-500$ & $500-800$ & $10-50$ & $50-100$ & $100-200$ & $200-500$ & $500-800$ \\
\hline & & 135.5 & 0.3 & 1.1 & 17.8 & & & 19.9 & 2.6 & 9.3 & 2.1 & 0 & 0.6 & 1.4 & 2.5 & 246.4 \\
\hline $112 \mathrm{a}$ & Alicante & 0 & 0.2 & 0 & 2.8 & 0 & 0 & 1.0 & 0.3 & 1.1 & 1.2 & 0 & 2.7 & 0 & 1.1 & 0 \\
\hline $113 \mathrm{a}$ & Catalan Sea & 0 & 1.5 & 0.8 & 0 & 0 & 0.3 & 0.1 & 0.2 & 0.7 & 0 & 0 & 0.6 & 0.4 & 0.6 & 0.9 \\
\hline $114 \mathrm{a}$ & W Morocco & * & * & * & * & * & * & * & $*$ & * & * & * & * & $*$ & * & * \\
\hline $114 \mathrm{~b}$ & E Morocco & $*$ & $*$ & $*$ & $*$ & $*$ & $*$ & * & $*$ & * & $*$ & $*$ & $*$ & $*$ & * & $*$ \\
\hline $121 \mathrm{a}$ & W Gulf of Lions & 0 & 0 & 0 & 3.1 & 1.9 & 0 & 0.1 & 2.0 & 0 & 1.2 & 0.1 & 0 & 6.8 & 0 & 0 \\
\hline $121 b$ & E Gulf of Lions & 0 & 0.2 & 0.3 & 34.0 & 0 & 0 & 0 & 0 & 51.7 & 28.8 & 0 & 0.2 & 0.2 & 0 & 56.1 \\
\hline $131 \mathrm{a}$ & NE Corsica & $*$ & 0 & 0 & 0.7 & 3.5 & $*$ & 0 & 0 & 0.8 & 0 & $*$ & 0 & 0 & 0.5 & 2.2 \\
\hline $131 b$ & SE Corsica & * & 0 & 0 & 0 & 0 & * & 0 & 0 & 0 & 0 & * & 4.3 & 0 & 0.6 & 0.7 \\
\hline $132 \mathrm{a}$ & N Ligurian Sea & 0 & 0 & 0 & 2.0 & 0.3 & 0 & 0 & 0 & 0.6 & 0.6 & 0 & 0 & 0 & 14.9 & 7.9 \\
\hline $132 b$ & E Ligurian Sea & 0.1 & 0.2 & 0.1 & 25.5 & 11.6 & 0 & 0 & 1.0 & 2.7 & 46.2 & 24.5 & 0.3 & 2.1 & 2.4 & 2.6 \\
\hline $132 \mathrm{c}$ & N Tyrrhenian & 1 & 0 & 2.8 & 0.2 & 0.4 & 0 & 0 & 0.2 & 1.0 & 0.6 & 0 & 0 & 0.8 & 0.7 & 0.2 \\
\hline $132 \mathrm{~d}$ & C Tyrrhenian & 0 & 0 & 0 & 0.3 & 0.6 & 0 & 0 & 0 & 0.4 & 1.0 & 0 & 0 & 0.6 & 0.6 & 0 \\
\hline $133 \mathrm{a}$ & SE Sardinia & 0 & 0 & 0 & 0 & 0 & 0 & 0 & 0 & 0 & 0 & 0 & 0 & 0 & 0 & 0 \\
\hline $133 b$ & NE Sardinia & 0 & 0 & 0 & 0 & 0 & 0 & 0 & 0 & 0 & 0.8 & 0 & 0 & 0 & 0 & 0 \\
\hline $133 \mathrm{c}$ & N Sardinia & 0 & 0 & 0 & 0 & 0 & 0 & 0 & 0 & 3.4 & 4.0 & 0 & 0 & 0 & 0 & 0 \\
\hline $133 d$ & NW Sardinia & 0 & 0 & 0 & 0 & 0 & * & 0 & 0 & 0 & 13.5 & 0 & 0 & 2.3 & 0 & 0 \\
\hline $133 \mathrm{e}$ & W Sardinia & 0 & * & 0 & 0 & 0 & 0 & 0 & 0 & 2.0 & 0 & 0 & 0 & 0 & 0 & 0 \\
\hline $133 \mathrm{f}$ & SW Sardinia & 0 & 0 & 0 & 0.4 & 0 & 0 & 1.8 & 0 & 1.2 & 9.3 & 18.7 & 1.6 & 0.3 & 0 & 29.5 \\
\hline $133 \mathrm{~g}$ & S Sardinia & 0 & 0 & 0 & 0 & 0 & 0 & * & 0 & 0 & 0.9 & 0 & 0 & 0 & 88.1 & 0 \\
\hline $134 a$ & SE Tyrrhenian & 0 & 0 & 0.4 & 0.5 & 0.3 & 0.9 & 0 & 0.1 & 0.9 & 5.8 & 0.4 & 0.4 & 0.5 & 0 & 0 \\
\hline $134 b$ & SW Tyrrhenian & 0 & 13.3 & 1.4 & 2.5 & 0.4 & 0.6 & 0 & 0.1 & 2.6 & 1.2 & 0 & 24.8 & 5.2 & 1.0 & 0 \\
\hline $134 \mathrm{c}$ & Sicilian Chan. & 0 & 0 & 0 & 0.3 & 0.3 & 0 & 0 & 0.8 & 0.7 & 0 & 0 & 0.3 & 1.7 & 0.5 & 0 \\
\hline $211 \mathrm{a}$ & N Adriatic Sea & 0 & 0 & * & $*$ & $*$ & 0 & 0 & * & * & * & 0 & 0.1 & $*$ & $*$ & * \\
\hline $211 b$ & Central Adriatic & 0 & 0 & 0 & 0 & 0 & 0 & 0 & 0 & 0.2 & 0 & 0 & 0 & 0.1 & 0.1 & 0 \\
\hline $211 \mathrm{c}$ & N Adriatic-Slov & $*$ & $*$ & $*$ & $*$ & $*$ & 0 & * & $*$ & * & * & 0 & $*$ & $*$ & $*$ & * \\
\hline $211 d$ & NE Adri Croatia & * & * & * & * & * & * & * & * & * & * & 0 & 0.4 & 0.2 & 0 & * \\
\hline $221 \mathrm{a}$ & E Sicily & 0 & 0 & 0 & 0.8 & 0 & 0 & 0 & 0 & 0.6 & 0 & 0 & 0 & 0 & 0 & 0 \\
\hline $221 b$ & NW Ionian Sea & 0 & 0 & 0 & 0.4 & 0 & 0 & 0 & 0 & 0 & 0.1 & 0 & 0 & 0 & 0 & 0 \\
\hline $221 \mathrm{c}$ & N Ionian Sea & 0 & 0 & 0 & 0 & 0 & 0 & 0 & 0 & 0 & 0 & 0 & 0 & 0 & 0 & 0 \\
\hline $221 d$ & N Ionian Sea & $*$ & 0 & 0 & 0 & 0 & 0 & 0 & 0 & 0 & 0 & 0 & 0 & 0 & 0 & 0.2 \\
\hline $221 \mathrm{e}$ & SW Adriatic & 0 & 0 & 0.3 & 0.7 & 0 & * & 0 & 0 & 0 & 0.6 & * & 0 & 0 & 0 & 0 \\
\hline $221 \mathrm{f}$ & SW Adriatic & 0 & 0 & 0 & 0 & 0 & 0 & 0 & 0 & 0 & 0 & 0 & 0 & 0 & 0 & 0 \\
\hline $221 \mathrm{~g}$ & SW Adriatic & 0.1 & 0 & 0 & $*$ & 0 & 0 & 0 & 0 & $*$ & 0 & 0 & 0 & 0 & $*$ & 0 \\
\hline $221 \mathrm{~h}$ & SW Adriatic & 0 & 0 & 0 & 0 & 3.6 & 0 & 0 & 0 & 0.6 & 0 & 0 & 0 & 0 & 0.3 & 0 \\
\hline $221 \mathrm{i}$ & SE Adriatic & * & * & * & * & $*$ & * & * & $*$ & * & * & 0 & 0 & 0.7 & 0.1 & 0 \\
\hline $222 \mathrm{a}$ & E Ionian Sea & 0 & 0.1 & 0 & 0 & 0 & 0 & 0 & 0 & 0 & 0 & 0.1 & 0.5 & 0 & 0.1 & 0 \\
\hline $223 a$ & Argosaronikos & 0 & 1.5 & 1.3 & 12.9 & 0 & 0.2 & 0 & 0.3 & 0 & 0 & 0 & 0.1 & 0 & 0 & 0 \\
\hline $224 a$ & N Aegean Sea & 1.5 & 0 & 0.1 & 0.3 & 1.7 & 0 & 0 & 0 & 0 & 0 & 0 & 0 & 0 & 3.3 & 0.3 \\
\hline $225 \mathrm{a}$ & S Aegean Sea & 0 & 9.5 & 0 & 0.9 & 0.9 & 0.3 & 0.1 & 0.3 & 2.5 & 2.5 & 0 & 0 & 0 & 3.8 & 0.6 \\
\hline Sector code & Sector & $10-50$ & & $\begin{array}{l}1997 \\
\text { epth ( } \\
00-200\end{array}$ & & & $10-50$ & $50-100$ & $\begin{array}{l}1998 \\
\text { Depth } \\
100-200\end{array}$ & $\begin{array}{l}8 \\
(\mathrm{~m}) \\
200-500\end{array}$ & & $10-50$ & ${ }_{50-100}^{\mathrm{D}}$ & $\begin{array}{r}1999 \\
\text { Depth (1 }\end{array}$ & $\begin{array}{l}\text { m) } \\
200-500\end{array}$ & $500-800$ \\
\hline Sector code & Sector & $10-30$ & $50-100$ & & & $500-800$ & $10-30$ & $30-100$ & $0-2002$ & & $500-800$ & $10-50$ & & & & \\
\hline $111 \mathrm{a}$ & Alborán Sea & 0 & 1.1 & 5.4 & 5.8 & 5.7 & 6.9 & 8.9 & 87.8 & 0.5 & 0.4 & 0 & 0 & 0.3 & 0.8 & 1.5 \\
\hline $112 \mathrm{a}$ & Alicante & 0 & 7.1 & 0.3 & 3.2 & 1.5 & 0 & 5.6 & 0 & 0 & 0 & 0.2 & 7.5 & 0 & 0 & 0 \\
\hline $113 a$ & Catalan Sea & 0 & 0.7 & 0.9 & 2.8 & 1.0 & 0 & 0.2 & 0.4 & $*$ & 2.6 & 0 & 0 & 0.6 & 0.4 & 0 \\
\hline $114 \mathrm{a}$ & W Morocco & $*$ & * & * & * & * & * & * & * & * & * & * & 0 & 10.2 & 2.2 & 0 \\
\hline $114 b$ & E Morocco & * & $*$ & * & * & $*$ & * & * & * & $*$ & * & 0 & 0 & 0.5 & 1.5 & 2.2 \\
\hline $121 \mathrm{a}$ & W Gulf of Lions & 0.1 & 1.5 & 0 & 0 & 0 & 0 & 0 & 0.7 & 0 & 1.2 & 2.2 & 0.3 & 0 & 0.8 & 0 \\
\hline $121 b$ & E Gulf of Lions & 0 & 0.1 & 0.2 & 1.1 & 0 & 0 & 0 & 1.2 & 129.0 & 4.8 & 6.7 & 1.2 & 1.5 & 113.5 & * \\
\hline $131 \mathrm{a}$ & NE Corsica & * & 0 & $*$ & 0 & 0 & $*$ & 0 & 0 & 0.2 & 0 & $*$ & 0 & 0 & 0.4 & 2.2 \\
\hline $131 b$ & SE Corsica & * & 0 & 0 & 0.9 & $*$ & * & 0 & 0 & 0.4 & 591.1 & * & 0 & 0 & 0 & 108.4 \\
\hline $132 \mathrm{a}$ & N Ligurian Sea & 0 & 0 & 0 & 3.0 & 1.6 & 0 & 0.7 & 0 & 100.7 & 0.7 & 0 & 1.4 & 0.8 & 6.8 & 8.6 \\
\hline $132 b$ & E Ligurian Sea & 1.3 & 0.2 & 8.3 & 13.0 & 0 & 0 & 0 & 0 & 5.2 & 2.9 & 3.3 & 0.2 & 1.5 & 10.7 & 2.0 \\
\hline $132 \mathrm{c}$ & $\mathrm{N}$ Tyrrhenian & 0.4 & 0 & 0 & 0.9 & 0.5 & 0 & 0 & 0.7 & 0 & 0.6 & 0 & 0 & 0.2 & 0.4 & 0.6 \\
\hline $132 \mathrm{~d}$ & C Tyrrhenian & 0 & 0 & 0.2 & 0 & 0 & 0 & 0 & 0.3 & 0.3 & 0.3 & 4.2 & 0.3 & 0.3 & 1.1 & 0.6 \\
\hline $133 a$ & SE Sardinia & 0 & 0 & 0 & 0.6 & 1.4 & 0 & 0 & 0 & 0 & 2.2 & 0 & 0 & 0 & 0 & 4.3 \\
\hline $133 b$ & NE Sardinia & 0 & 0 & 0 & 0 & 0 & 0 & 0 & 0 & 1.4 & 1.1 & 0 & 0 & 0 & 0.6 & 0.4 \\
\hline $133 \mathrm{c}$ & N Sardinia & 0 & 0 & 0 & 0 & 2.3 & 0 & 0 & 0 & 0.9 & 4.4 & 0 & 0 & 0 & 0 & 2.2 \\
\hline $133 d$ & NW Sardinia & 0 & 0 & 0 & 0 & 0 & 0 & 0 & 0 & 0 & 0.9 & 0 & 0 & 1.9 & 1.0 & 0 \\
\hline $133 \mathrm{e}$ & W Sardinia & 0 & 0 & 0.4 & 0 & 0 & 0 & 0 & 2.1 & 2.9 & 2.0 & 0 & 0 & 0.7 & 0.5 & 4.9 \\
\hline $133 \mathrm{f}$ & SW Sardinia & 0 & 0.2 & 0 & 0.2 & 0.3 & 0 & 0 & 0.2 & 2.8 & 2.4 & 1.5 & 7.5 & 1.4 & 1.2 & 11.3 \\
\hline $133 \mathrm{~g}$ & S Sardinia & 0 & 0 & 0.8 & 0 & 0 & 0 & 0 & 0 & 1.0 & 0.2 & 0 & 0 & 0 & 8.1 & 7.2 \\
\hline $134 a$ & SE Tyrrhenian & 0.5 & 0 & 0.1 & 0.5 & 0.5 & 1.2 & 0 & 0.3 & 0.8 & 0.5 & 0.2 & 0.4 & 0.4 & 1.5 & 0.6 \\
\hline $134 b$ & SW Tyrrhenian & 0 & 32.7 & 1.7 & 0 & 0.3 & 0 & 15.1 & 0 & 1.1 & 0 & 0 & 43.7 & 15.9 & 2.3 & 0 \\
\hline
\end{tabular}


TABLE 6 (Cont.). - Pagellus bogaraveo: mean biomass $\left(\mathrm{kg} / \mathrm{km}^{2}\right)$ estimated from the MEDITS trawl surveys by depth stratum, geographical sector and year (1994-1999). Strata that were not sampled are indicated by '*'. Values higher than $10 \mathrm{~kg} / \mathrm{km}^{2}$ are presented in bold.

\begin{tabular}{|c|c|c|c|c|c|c|c|c|c|c|c|c|c|c|c|c|}
\hline \multirow{3}{*}{$\frac{\text { Sector code }}{134 \mathrm{c}}$} & \multirow{3}{*}{$\begin{array}{l}\text { Sector } \\
\text { Sicilian Chan. }\end{array}$} & \multicolumn{5}{|c|}{$\begin{array}{c}1997 \\
\text { Depth (m) }\end{array}$} & \multicolumn{5}{|c|}{$\begin{array}{c}1998 \\
\text { Depth (m) }\end{array}$} & \multicolumn{5}{|c|}{$\begin{array}{c}1999 \\
\text { Depth (m) }\end{array}$} \\
\hline & & $10-50$ & $50-100$ & $0-200$ & -500 & $500-800$ & $10-50$ & $50-100$ & $00-200$ & $200-500$ & $0-800$ & $10-50$ & $50-100$ & & $200-500$ & $500-800$ \\
\hline & & 0 & 0 & 0.1 & 0 & 0.1 & 0 & 0 & 0.1 & 0.1 & 0.3 & 0 & 0.4 & 0.2 & 0.2 & 0 \\
\hline $211 \mathrm{a}$ & N Adriatic Sea & 0 & 0 & * & * & * & 0 & 0 & $*$ & * & $*$ & 0.1 & 0 & * & $*$ & * \\
\hline $211 \mathrm{~b}$ & Central Adriatic & 0 & 0 & 0.1 & 0.5 & 0 & 0 & 0 & 0.1 & 0 & $*$ & 1 & 0 & 0 & 0 & $*$ \\
\hline $211 \mathrm{c}$ & N Adriatic-Slov & 0 & * & * & * & * & 0 & * & $*$ & $*$ & $*$ & 0 & * & * & * & * \\
\hline $211 \mathrm{~d}$ & NE Adri Croatia & 0 & 0.6 & 0.2 & 0 & * & 0 & 0.2 & 0 & 0.9 & * & 0 & * & * & * & * \\
\hline $221 \mathrm{a}$ & E Sicily & 0 & 0 & 0 & 0 & 2.3 & 0 & 0 & 0 & 0 & 0 & 0 & 0 & 0 & 0 & 0 \\
\hline $221 b$ & NW Ionian Sea & 0 & 0 & 0 & 0.6 & 0.2 & 0 & 0 & 0 & 0 & 0 & 0 & 0 & 0 & 0 & 0 \\
\hline $221 \mathrm{c}$ & N Ionian Sea & 0 & 0 & 0 & 0 & 0 & 0 & 0 & 0 & 0 & 0 & 0 & 0 & 0 & 0 & 0 \\
\hline $221 \mathrm{~d}$ & N Ionian Sea & 0 & 0 & 0 & 0 & 0.4 & 0 & 0 & 0 & 0 & 0 & 0 & 0 & 0 & 0 & 0 \\
\hline $221 \mathrm{e}$ & SW Adriatic & * & 0 & 0 & 0 & 0 & * & 0 & 0 & 0.3 & 0 & $*$ & 0 & 0 & 0.3 & 0 \\
\hline $221 \mathrm{f}$ & SW Adriatic & 0 & 0 & 0 & 0 & 1.1 & 0 & 0 & 0 & 0 & 0 & 1.5 & 0 & 0 & 0 & 0 \\
\hline $221 \mathrm{~g}$ & SW Adriatic & 0 & 0 & 0 & * & 0 & 0 & 0 & 0 & * & 7.3 & 0.1 & 0 & 0 & * & 0 \\
\hline $221 \mathrm{~h}$ & SW Adriatic & 0 & 0 & 0 & 0.3 & 0 & 0 & 0 & 0 & 0 & 0 & 0.1 & 0 & 0 & 0 & 3.7 \\
\hline $221 \mathrm{i}$ & SE Adriatic & 0 & 0 & 0.2 & 0.3 & 0.9 & 0 & 0 & 0 & 0 & 1.6 & 1.6 & 0 & 0 & 0.3 & 0 \\
\hline $222 \mathrm{a}$ & E Ionian Sea & 0 & 0 & 0 & 0.5 & 0 & 0 & 0.2 & 0 & 5.7 & 1.0 & 0 & 0 & 0.3 & 7.7 & 28.5 \\
\hline $223 \mathrm{a}$ & Argosaronikos & 7.5 & 0 & 0.5 & 0.1 & 0 & 0 & 1.8 & 0.1 & 0.9 & 0.2 & 0 & 0.2 & 0.2 & 0.3 & 0 \\
\hline $224 a$ & N Aegean Sea & 0 & 1.4 & 6.1 & 4.6 & 1.8 & 0 & 0 & 3.9 & 8.7 & 3.7 & 0 & 0.2 & 0 & 21.1 & 1.4 \\
\hline $225 \mathrm{a}$ & S Aegean Sea & 0 & 0.9 & 0 & 9.2 & 0.4 & 0 & 0 & 0 & 18.8 & 7.9 & 0 & 0 & 0.1 & 9.2 & 10.4 \\
\hline
\end{tabular}

TABLE 7. - Pagellus bogaraveo: mean density (in number of individuals $/ \mathrm{km}^{2}$ ) estimated from the MEDITS trawl surveys by depth stratum, geographical sector and year (1994-1999). Strata that were not sampled are indicated by '*'. Values higher than 200 individuals $/ \mathrm{km}^{2}$ are presented in bold.

\begin{tabular}{|c|c|c|c|c|c|c|c|c|c|c|c|c|c|c|c|c|}
\hline \multirow{2}{*}{$\begin{array}{l}\text { Sector code } \\
111 \mathrm{a}\end{array}$} & \multirow{2}{*}{$\begin{array}{l}\text { Sector } \\
\text { Alborán Sea }\end{array}$} & \multicolumn{5}{|c|}{$\begin{array}{c}1994 \\
\text { Depth (m) }\end{array}$} & \multicolumn{5}{|c|}{$\begin{array}{c}1995 \\
\text { Depth (m) }\end{array}$} & \multicolumn{5}{|c|}{$\begin{array}{c}1996 \\
\text { Depth (m) }\end{array}$} \\
\hline & & 4545 & 5 & 7 & 120 & 82 & 0 & 409 & 44 & 58 & 9 & 0 & 22 & 30 & 16 & 338 \\
\hline $112 \mathrm{a}$ & Alicante & 0 & 4 & 0 & 20 & 0 & 0 & 14 & 5 & 5 & 5 & 0 & 47 & 0 & 6 & 0 \\
\hline $113 a$ & Catalan Sea & 0 & 28 & 12 & 0 & 0 & 8 & 3 & 2 & 2 & 0 & 0 & 12 & 6 & 2 & 3 \\
\hline $114 a$ & W Morocco & * & $*$ & $*$ & $*$ & * & * & * & $*$ & * & * & * & $*$ & $*$ & $*$ & * \\
\hline $114 b$ & E Morocco & * & * & * & $*$ & $*$ & * & * & $*$ & * & $*$ & $*$ & $*$ & $*$ & $*$ & * \\
\hline $121 \mathrm{a}$ & W Gulf of Lions & 0 & 0 & 0 & 2 & 4 & 0 & 1 & 28 & 0 & 2 & 3 & 0 & 100 & 0 & 0 \\
\hline $121 b$ & E Gulf of Lions & 0 & 2 & 2 & 76 & 0 & 0 & 0 & 0 & 103 & 32 & 0 & 2 & 2 & 0 & 35 \\
\hline $131 \mathrm{a}$ & NE Corsica & * & 0 & 0 & 6 & 3 & * & 0 & 0 & 6 & 0 & * & 0 & 0 & 2 & 3 \\
\hline $131 \mathrm{~b}$ & SE Corsica & * & 0 & 0 & 0 & 0 & $*$ & 0 & 0 & 0 & 0 & $*$ & 173 & 0 & 3 & 5 \\
\hline $132 \mathrm{a}$ & N Ligurian Sea & 0 & 0 & 0 & 9 & 1 & 0 & 0 & 0 & 4 & 2 & 0 & 0 & 0 & 147 & 4 \\
\hline $132 b$ & E Ligurian Sea & 8 & 3 & 1 & 32 & 11 & 0 & 0 & 14 & 9 & 37 & 1115 & 6 & 38 & 20 & 2 \\
\hline $132 \mathrm{c}$ & N Tyrrhenian & 133 & 0 & 22 & 2 & 1 & 0 & 0 & 2 & 6 & 3 & 0 & 0 & 10 & 6 & 1 \\
\hline $132 d$ & C Tyrrhenian & 0 & 0 & 0 & 3 & 1 & 0 & 0 & 0 & 2 & 3 & 0 & 0 & 18 & 5 & 0 \\
\hline $133 a$ & SE Sardinia & 0 & 0 & 0 & 0 & 0 & 0 & 0 & 0 & 0 & 0 & 0 & 0 & 0 & 0 & 0 \\
\hline $133 b$ & NE Sardinia & 0 & 0 & 0 & 0 & 0 & 0 & 0 & 0 & 0 & 3 & 0 & 0 & 0 & 0 & 0 \\
\hline $133 \mathrm{c}$ & N Sardinia & 0 & 0 & 0 & 0 & 0 & 0 & 0 & 0 & 15 & 20 & 0 & 0 & 0 & 0 & 0 \\
\hline $133 d$ & NW Sardinia & 0 & 0 & 0 & 0 & 0 & * & 0 & 0 & 0 & 42 & 0 & 0 & 6 & 0 & 0 \\
\hline $133 \mathrm{e}$ & W Sardinia & 0 & * & 0 & 0 & 0 & 0 & 0 & 0 & 7 & 0 & 0 & 0 & 0 & 0 & 0 \\
\hline $133 \mathrm{f}$ & SW Sardinia & 0 & 0 & 0 & 3 & 0 & 0 & 6 & 0 & 10 & 22 & 349 & 26 & 2 & 0 & 34 \\
\hline $133 \mathrm{~g}$ & S Sardinia & 0 & 0 & 0 & 0 & 0 & 0 & * & 0 & 0 & 4 & 0 & 0 & 0 & 35 & 0 \\
\hline $134 a$ & SE Tyrrhenian & 0 & 0 & 10 & 6 & 1 & 102 & 0 & 2 & 6 & 28 & 168 & 7 & 11 & 0 & 0 \\
\hline $134 \mathrm{~b}$ & SW Tyrrhenian & 0 & 284 & 37 & 43 & 2 & 6 & 0 & 3 & 25 & 6 & 0 & 617 & 114 & 8 & 0 \\
\hline $134 \mathrm{c}$ & Sicilian Chan. & 0 & 0 & 0 & 3 & 2 & 0 & 0 & 15 & 4 & 0 & 0 & 5 & 25 & 2 & 0 \\
\hline $211 \mathrm{a}$ & N Adriatic Sea & 0 & 0 & * & $*$ & * & 0 & 0 & * & * & * & 0 & 2 & * & $*$ & $*$ \\
\hline $211 b$ & Central Adriatic & 0 & 0 & 0 & 0 & 0 & 0 & 0 & 0 & 2 & 0 & 0 & 0 & 1 & 2 & 0 \\
\hline $211 \mathrm{c}$ & N Adriatic-Slov & $*$ & $*$ & $*$ & $*$ & * & 0 & * & $*$ & * & $*$ & 0 & $*$ & * & $*$ & $*$ \\
\hline 211d & NE Adri Croatia & * & * & * & * & * & * & * & * & * & * & 2 & 18 & 5 & 0 & $*$ \\
\hline $221 \mathrm{a}$ & E Sicily & 0 & 0 & 0 & 12 & 0 & 0 & 0 & 0 & 3 & 0 & 0 & 0 & 0 & 0 & 0 \\
\hline $221 b$ & NW Ionian Sea & 0 & 0 & 0 & 5 & 0 & 0 & 0 & 0 & 0 & 1 & 0 & 0 & 0 & 0 & 0 \\
\hline $221 \mathrm{c}$ & N Ionian Sea & 0 & 0 & 0 & 0 & 0 & 0 & 0 & 0 & 0 & 0 & 0 & 0 & 0 & 0 & 0 \\
\hline 221d & N Ionian Sea & * & 0 & 0 & 0 & 0 & 0 & 0 & 0 & 0 & 0 & 0 & 0 & 0 & 0 & 1 \\
\hline $221 \mathrm{e}$ & SW Adriatic & 0 & 0 & 5 & 7 & 0 & * & 0 & 0 & 0 & 2 & * & 0 & 0 & 0 & 0 \\
\hline $221 f$ & SW Adriatic & 0 & 0 & 0 & 0 & 0 & 0 & 0 & 0 & 0 & 0 & 0 & 0 & 0 & 0 & 0 \\
\hline $221 \mathrm{~g}$ & SW Adriatic & 12 & 0 & 0 & $*$ & 0 & 0 & 0 & 0 & * & 0 & 0 & 0 & 0 & $*$ & 0 \\
\hline $221 \mathrm{~h}$ & SW Adriatic & 0 & 0 & 0 & 0 & 10 & 0 & 0 & 0 & 5 & 0 & 0 & 0 & 0 & 6 & 0 \\
\hline $221 \mathrm{i}$ & SE Adriatic & * & * & * & * & * & * & * & $*$ & * & * & 0 & 0 & 10 & 3 & 0 \\
\hline $222 \mathrm{a}$ & E Ionian Sea & 0 & 9 & 0 & 0 & 0 & 0 & 0 & 0 & 0 & 0 & 13 & 28 & 0 & 3 & 0 \\
\hline $223 a$ & Argosaronikos & 0 & 21 & 13 & 129 & 0 & 20 & 0 & 5 & 0 & 0 & 0 & 8 & 0 & 0 & 0 \\
\hline $224 a$ & N Äegean Sea & 137 & 0 & 5 & 5 & 8 & 0 & 0 & 0 & 0 & 0 & 3 & 0 & 0 & 44 & 3 \\
\hline $225 \mathrm{a}$ & S Aegean Sea & 0 & 242 & 0 & 13 & 5 & 27 & 3 & 6 & 38 & 16 & 0 & 0 & 0 & 31 & 5 \\
\hline
\end{tabular}


TABle 7 (Cont.). - Pagellus bogaraveo: mean density (in number of individuals $/ \mathrm{km}^{2}$ ) estimated from the MEDITS trawl surveys by depth stratum, geographical sector and year (1994-1999). Strata that were not sampled are indicated by '*'. Values higher than 200 individuals /km² are presented in bold.

\begin{tabular}{|c|c|c|c|c|c|c|c|c|c|c|c|c|c|c|c|c|}
\hline \multirow{2}{*}{$\begin{array}{l}\text { Sector code } \\
111 \mathrm{a}\end{array}$} & \multirow{2}{*}{$\begin{array}{l}\text { Sector } \\
\text { Alborán Sea }\end{array}$} & \multicolumn{5}{|c|}{$\begin{array}{c}1997 \\
\text { Depth (m) }\end{array}$} & \multicolumn{5}{|c|}{$\begin{array}{c}1998 \\
\text { Depth (m) }\end{array}$} & \multicolumn{5}{|c|}{$\begin{array}{c}1999 \\
\text { Depth (m) }\end{array}$} \\
\hline & & 0 & 21 & 43 & 39 & 16 & 116 & 175 & 1396 & 3 & 2 & 0 & 0 & 4 & 4 & 4 \\
\hline $112 \mathrm{a}$ & Alicante & 0 & 221 & 8 & 31 & 8 & 0 & 147 & 0 & 0 & 0 & 6 & 144 & 0 & 0 & 0 \\
\hline $113 a$ & Catalan Sea & 0 & 13 & 12 & 9 & 3 & 0 & 3 & 6 & $*$ & 10 & 0 & 0 & 6 & 2 & 0 \\
\hline $114 \mathrm{a}$ & W Morocco & $*$ & $*$ & $*$ & $*$ & $*$ & $*$ & $*$ & $*$ & $*$ & $*$ & $*$ & 0 & 160 & 14 & 0 \\
\hline $114 b$ & E Morocco & $*$ & $*$ & * & $*$ & $*$ & $*$ & $*$ & $*$ & $*$ & $*$ & 0 & 0 & 12 & 4 & 5 \\
\hline $121 \mathrm{a}$ & W Gulf of Lions & 2 & 22 & 0 & 0 & 0 & 0 & 0 & 5 & 0 & 2 & 39 & 4 & 0 & 2 & 0 \\
\hline $121 b$ & E Gulf of Lions & 0 & 1 & 2 & 6 & 0 & 0 & 0 & 15 & 176 & 8 & 111 & 7 & 16 & 179 & $*$ \\
\hline $131 \mathrm{a}$ & NE Corsica & $*$ & 0 & * & 0 & 0 & $*$ & 0 & 0 & 3 & 0 & $*$ & 0 & 0 & 2 & 8 \\
\hline $131 b$ & SE Corsica & $*$ & 0 & 0 & 9 & $*$ & $*$ & 0 & 0 & 3 & 399 & $*$ & 0 & 0 & 0 & 104 \\
\hline $132 \mathrm{a}$ & N Ligurian Sea & 0 & 0 & 0 & 21 & 1 & 0 & 21 & 0 & 158 & 1 & 0 & 29 & 37 & 17 & 13 \\
\hline $132 b$ & E Ligurian Sea & 150 & 3 & 108 & 18 & 0 & 0 & 0 & 0 & 23 & 2 & 549 & 3 & 24 & 10 & 2 \\
\hline $132 \mathrm{c}$ & $\mathrm{N}$ Tyrrhenian & 5 & 0 & 0 & 4 & 2 & 0 & 0 & 6 & 0 & 3 & 0 & 0 & 2 & 3 & 2 \\
\hline $132 d$ & C Tyrrhenian & 0 & 0 & 1 & 0 & 0 & 0 & 0 & 3 & 2 & 1 & 75 & 3 & 3 & 5 & 2 \\
\hline $133 a$ & SE Sardinia & 0 & 0 & 0 & 4 & 4 & 0 & 0 & 0 & 0 & 8 & 0 & 0 & 0 & 0 & 18 \\
\hline $133 b$ & NE Sardinia & 0 & 0 & 0 & 0 & 0 & 0 & 0 & 0 & 10 & 6 & 0 & 0 & 0 & 4 & 2 \\
\hline $133 \mathrm{c}$ & N Sardinia & 0 & 0 & 0 & 0 & 10 & 0 & 0 & 0 & 6 & 22 & 0 & 0 & 0 & 0 & 21 \\
\hline $133 d$ & NW Sardinia & 0 & 0 & 0 & 0 & 0 & 0 & 0 & 0 & 0 & 3 & 0 & 0 & 13 & 5 & 0 \\
\hline $133 \mathrm{e}$ & W Sardinia & 0 & 0 & 4 & 0 & 0 & 0 & 0 & 26 & 17 & 11 & 0 & 0 & 5 & 5 & 22 \\
\hline $133 f$ & SW Sardinia & 0 & 6 & 0 & 1 & 1 & 0 & 0 & 2 & 13 & 5 & 32 & 85 & 9 & 6 & 36 \\
\hline $133 \mathrm{~g}$ & S Sardinia & 0 & 0 & 11 & 0 & 0 & 0 & 0 & 0 & 6 & 2 & 0 & 0 & 0 & 3 & 24 \\
\hline $134 \mathrm{a}$ & SE Tyrrhenian & 38 & 0 & 2 & 5 & 4 & 44 & 0 & 5 & 5 & 2 & 6 & 17 & 11 & 7 & 2 \\
\hline $134 b$ & SW Tyrrhenian & 0 & 752 & 35 & 0 & 1 & 0 & 460 & 0 & 6 & 0 & 0 & 1048 & 384 & 15 & 0 \\
\hline $134 \mathrm{c}$ & Sicilian Chan. & 0 & 0 & 2 & 0 & 1 & 0 & 0 & 2 & 1 & 1 & 0 & 8 & 4 & 1 & 0 \\
\hline $211 \mathrm{a}$ & N Adriatic Sea & 0 & 0 & $*$ & $*$ & $*$ & 0 & 0 & $*$ & $*$ & $*$ & 4 & 0 & $*$ & $*$ & $*$ \\
\hline $211 b$ & Central Adriatic & 0 & 0 & 1 & 6 & 0 & 0 & 0 & 1 & 0 & $*$ & 46 & 0 & 0 & 0 & $*$ \\
\hline $211 \mathrm{c}$ & N Adriatic-Slov & 0 & $*$ & $*$ & $*$ & $*$ & 0 & $*$ & $*$ & $*$ & $*$ & 0 & $*$ & $*$ & $*$ & $*$ \\
\hline $211 d$ & NE Adri Croatia & 0 & 16 & 3 & 0 & $*$ & 0 & 6 & 1 & 10 & $*$ & 0 & $*$ & $*$ & $*$ & $*$ \\
\hline $221 \mathrm{a}$ & E Sicily & 0 & 0 & 0 & 0 & 11 & 0 & 0 & 0 & 0 & 0 & 0 & 0 & 0 & 0 & 0 \\
\hline $221 b$ & NW Ionian Sea & 0 & 0 & 0 & 8 & 1 & 0 & 0 & 0 & 0 & 0 & 0 & 0 & 0 & 0 & 0 \\
\hline $221 \mathrm{c}$ & N Ionian Sea & 0 & 0 & 0 & 0 & 0 & 0 & 0 & 0 & 0 & 0 & 0 & 0 & 0 & 0 & 0 \\
\hline $221 d$ & N Ionian Sea & 0 & 0 & 0 & 0 & 1 & 0 & 0 & 0 & 0 & 0 & 0 & 0 & 0 & 0 & 0 \\
\hline $221 \mathrm{e}$ & SW Adriatic & $*$ & 0 & 0 & 0 & 0 & $*$ & 0 & 0 & 2 & 0 & $*$ & 0 & 0 & 2 & 0 \\
\hline $221 \mathrm{f}$ & SW Adriatic & 0 & 0 & 0 & 0 & 11 & 0 & 0 & 0 & 0 & 0 & 105 & 0 & 0 & 0 & 0 \\
\hline $221 \mathrm{~g}$ & SW Adriatic & 0 & 0 & 0 & $*$ & 0 & 0 & 0 & 0 & $*$ & 32 & 12 & 0 & 0 & $*$ & 0 \\
\hline $221 \mathrm{~h}$ & SW Adriatic & 0 & 0 & 0 & 6 & 0 & 0 & 0 & 0 & 0 & 0 & 5 & 0 & 0 & 0 & 14 \\
\hline $221 \mathrm{i}$ & SE Adriatic & 0 & 0 & 5 & 4 & 4 & 0 & 0 & 0 & 0 & 4 & 131 & 2 & 0 & 3 & 0 \\
\hline $222 a$ & E Ionian Sea & 0 & 0 & 0 & 6 & 0 & 0 & 5 & 0 & 50 & 9 & 0 & 0 & 4 & 65 & 168 \\
\hline $223 a$ & Argosaronikos & 14 & 0 & 6 & 5 & 0 & 0 & 39 & 3 & 15 & 3 & 7 & 24 & 3 & 8 & 0 \\
\hline $224 a$ & N Aegean Sea & 0 & 41 & 131 & 57 & 19 & 0 & 0 & 91 & 109 & 36 & 0 & 4 & 0 & 312 & 16 \\
\hline $225 a$ & S Aegean Sea & 0 & 8 & 0 & 115 & 2 & 0 & 0 & 0 & 242 & 63 & 0 & 0 & 2 & 95 & 95 \\
\hline
\end{tabular}

\section{DISCUSSION}

All the three species of Pagellus were widely distributed throughout the sampling area. However, $P$. erythrinus was the species of the genus most frequently occurring in the samples, even though its preferential habitat is more restricted to the inshore waters (continental shelf) compared with $P$. acarne and especially $P$. bogaraveo. This outcome could mean that at the mesoscale level $P$. erythrinus is more extensively distributed with less patchy aggregations than the other two species or that its behaviour is less gregarious. However, this hypothesis needs to be tested by further specific analysis, like for example the geostatisical study of the spatial structure of abundance. Moreover, other factors, such as the availability of the different species to the trawl (i.e. change of habitat in relation with increasing size, daily vertical distribution and movements) or seasonal migration could affect the catch pattern in terms of yield and variability. Seasonal or local aggregations of any stock fractions could, thus, influence the catch abundance. With regard to $P$. bogaraveo a local distribution of larger fishes in the vicinity of offshore outcrops has been reported, for example, in the Ligurian Sea (e.g. Orsi Relini and Fida, 1992). Furthermore, wide ranging migrations from nursery areas to foraging grounds and viceversa seem to take place in the Atlantic (Desbrosses, 1932; Sánchez, 1983). This behavioural pattern of the Pagellus species can have influenced the sampling efficiency, as only a proportion of the stock could be available and accessible (Harden Jones, 1974) to the trawl. Indeed, along the Mediterranean 

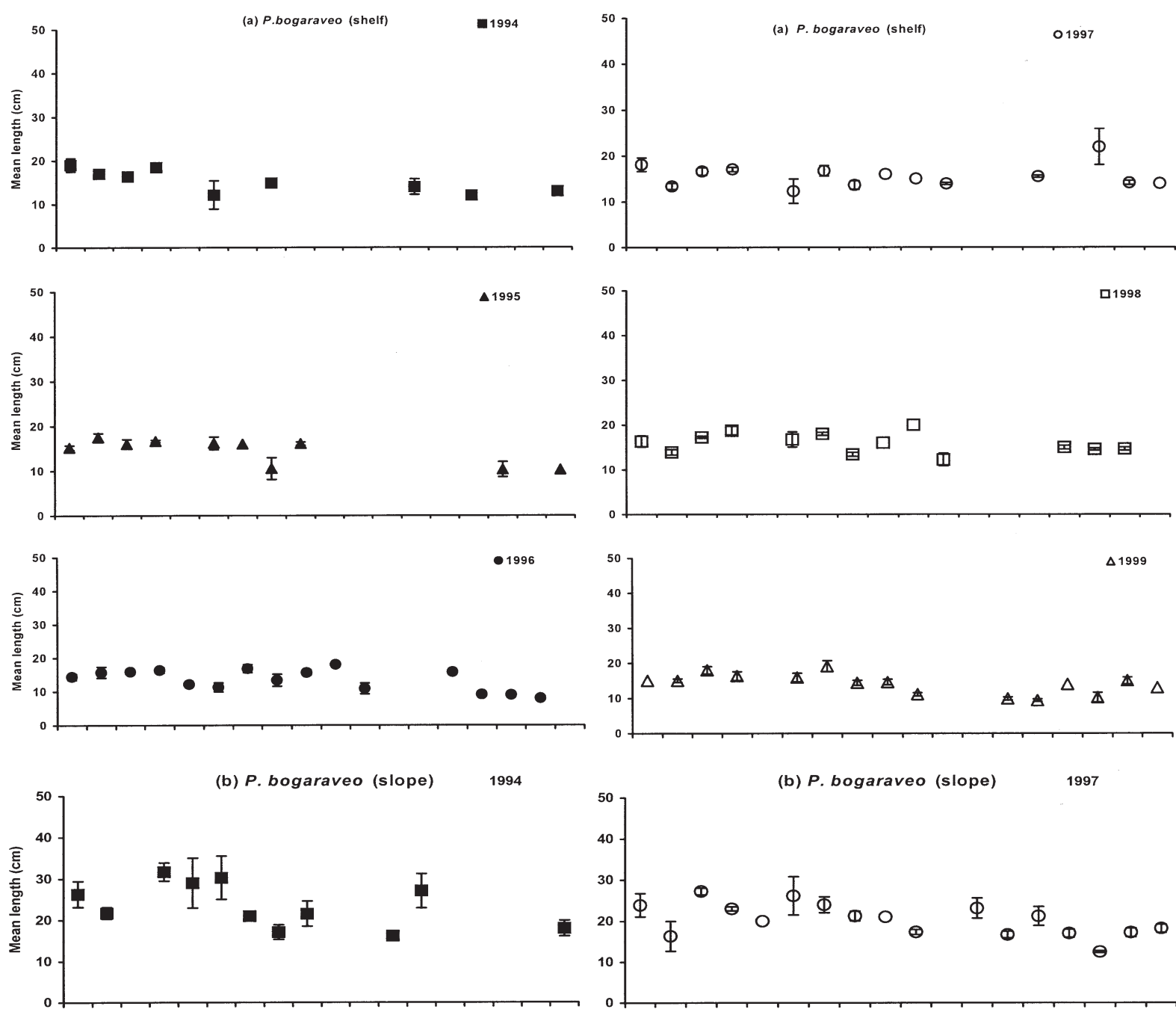

(b) P. bogaraveo (slope) 1997
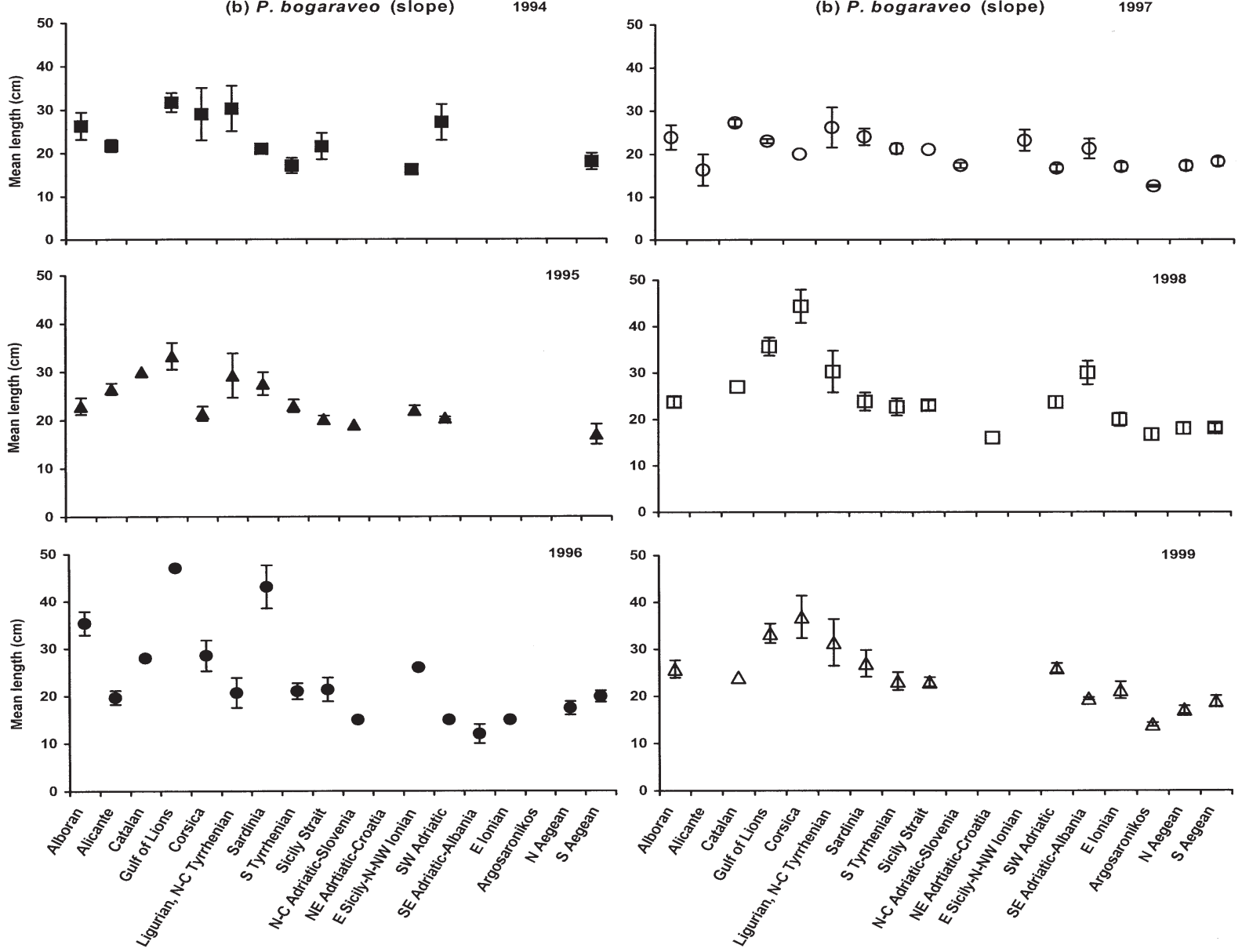

FIG. 3. - Mean total length $( \pm$ S.D.) of Pagellus bogaraveo on the shelf (a) and slope (b) by geographical region and year. 
and East Atlantic, the larger sizes of these fishes are mainly targeted by different gears, such as gill nets, longlines, and traps (e.g. Petrakis and Stergiou, 1996; Erzini et al., 1998; Pajuelo and Lorenzo, 1998; Sousa et al., 1999). Nevertheless, although the bias associated with the trawl survey indices makes it difficult to predict the absolute stock abundance, they can however be adopted as relative estimates of density and biomass related to a stock proportion. In this sense, especially for the juvenile fraction of the Pagellus populations, indices of relative abundance such as number of individuals or weight per square kilometre can be used as indicators of the geographical and inter-annual variations, provided that the trawl survey sampling scheme and protocols (e.g. stratification, gear, hauls, timing) are standardised.

The three Pagellus species showed different levels of abundance in the diverse geographical sectors, probably owing to the influence of several factors, such as the different fishing pressure in the area, the recruitment occurrence, or the specific environmental features. Although, for example, P. erythrinus was generally less abundant in the western sectors (from Alborán Sea to the Gulf of Lions), it was not possible to detect any gradient from west to east. Levels of density and biomass similar to the westward ones were also observed in central regions, such as in the North-Central Adriatic Sea and East Sicily-Ionian-South Adriatic. By contrast, high abundance indices were recorded in Corsica waters and not negligible values occurred in the first stratum of the Alborán Sea, as well. Low density and biomass were estimated in the South Tyrrhenian Sea due to the scarcity of $P$. erythinus in the northern side of the Sicily Strait sub-area. Conversely, this species seems to be important for the commercial fisheries in the southern side of this basin, and namely in the Gabes Gulf and along the Tunisian coasts (Jarboui et al., 1998). Relationships between levels of abundance and differences in fishing pressure among the geographical sectors are difficult to ascertain, due to the scarce information available on effective fishing effort and its distribution. However, the hypothesis of attributing, for example, the lower $P$. erythrinus abundance in the westernmost geographical sectors to a higher fishing effort than in the other zones is not consistent with the larger biomass and density of $P$. acarne in the same area. In fact, these two resources are supposed to be exposed to a comparable fishing pressure exerted by the same vessels operating on the shelf. On the other hand, a different occurrence of the recruits could essentially have affected the variations of the density indices among the geographical sectors, but did not substantially influence the relative biomass indices. Although the fishing pressure effect needs to be more deeply investigated, it seems that other factors, related to environmental conditions (oceanographic characteristics, prey availability and abundance, etc.) or fish movements, might play a role in determining the observed differences in abundance. Further studies are thus necessary to better clarify these aspects. The biomass indices of $P$. acarne and $P$. bogaraveo estimated in the Alborán Sea would indicate that these species are more abundant in the westernmost side of the Mediterranean. However, the remarkable density indices estimated in the eastern regions for $P$. acarne, in some cases (South Aegean in 1998) even higher than those observed in the Alborán Sea, suggest that this sparid does not exhibit a western preferential distribution.

The mean length of P. acarne and P. bogaraveo, estimated for shelf and slope, highlighted the occurrence of smaller individuals in the shallower stratum over most of the geographical range. This indicates, especially for $P$. bogaraveo, that migrations to deeper water take place with increasing length, as for example reported by Orsi Relini and Fida (1992) for the Ligurian Sea.

Small mean lengths of P. erythrinus were mainly observed in some areas (Ligurian-North Central Tyrrhenian Sea, South Tyrrhenian Sea, East Ionian, Argosaronikos) and a significant decreasing trend in the mean lengths from west to east was found. This condition also held for $P$. acarne and $P$. bogaraveo and in many cases was determined by the larger number of juveniles occurring in the central-eastern regions. The presence of $P$. erythrinus as small as 2$3 \mathrm{~cm}$ length is in accordance with a spring-summer spawning time (e.g. Larrañeta, 1964; Girardin and Quignard, 1985; Livadas, 1988; Mytilinéou, 1988). It is worth mentioning that in captive conditions with a natural thermo-photoperiod, $P$. erythrinus behaves as a sequential spawner, releasing egg batches from the middle of April to the middle of August (Spedicato et al., 1998). Thus, the recruitment could consist of several micro-cohorts entering the stock in successive phases. $P$. bogaraveo also seems to be characterised by a wide spawning period, lasting from November to May (e.g. Orsi Relini and Fida, 1992) and the presence of pelagic eggs of $P$. acarne is reported in summer and autumn in the Strait of Messina (De Gaetani, 1935). These fishes could also display a recruitment pattern similar to 
that supposed for $P$. erythrinus, with juveniles occurring in late spring-summer and autumn, according to the species. The decreasing trend of the mean lengths from west to east could also be attributed to possible differences in the growth pattern among the areas. However, this hypothesis requires further investigation. For P. erythrinus, for example, the available literature data (e.g. Larrañeta, 1964; Rijavec and Zupanovic, 1965; Andaloro and Prestipino Giarritta, 1985; Girardin and Quignard, 1985; Orsi Relini and Romeo, 1985; Livadas, 1988; Mytilineou, 1988) would indicate the existence of such a diversity. Nevertheless, the data are not strictly comparable, as different methods of estimation and measures (i.e. fork length, total length) were employed. On the other hand, the hypothesis of a higher fishing pressure determining lower mean lengths in the eastern sectors does not seem justified on the basis of the available information. Indeed, a proper analysis on this aspect would require specific historical data on geographical allocation of fishing effort and fishing intensity that are not available at present in all the Mediterranean regions, where abundance data have been collected since many years but no long data series of catch and effort exist. However, the indications from the long and short-term trend analyses of Mediterranean landings by Fiorentini et al. (1977) do not highlight an apparently different condition of the Pagellus species fishery from west to east. In addition, a landing and effort survey conducted in some sample ports of the Mediterranean (Anonymous, 2000), although with the limitation of the number of sampling sites, has not shown a higher fishing effort in the east side of the basin (Greek waters). Thus, the higher productivity of the western areas that are closest to the Atlantic inflow and whose shelves contribute more to the Mediterranean fishery production (in 1989: 4.78-8.15 versus $1.42-2.20$ tons $\cdot \mathrm{km}^{-2}$ of the east side; Caddy, 1997) could be a probable cause of mean lengths decreasing from west to east, although further investigations are necessary to confirm this hypothesis.

\section{REFERENCES}

Abella, A., R. Auteri, R. Baino, A. Lazzeretti, P. Righini, F. Serena, R. Silvestri, A. Voliani, and A. Zucchi. - 1997. Reclutamento di forme giovanili nella fascia costiera toscana. Biol. Mar. Medit., 4(1): 172-181.

Abellan, A. and B. Basurco. - 1999. Marine finfish species diversification: current situation and prospects in Mediterranean aquaculture. Opt. Médit., Sér. B: Etud. Rech., 24: 1-139.
Andaloro, F. - 1983a. About the catch, the diet, the reproduction, the size frequency and distribution of Pagellus acarne (Risso, 1826) in the Strait of Messina Area. Rapp. Comm. int. Mer Médit., 28(5): 33-37.

Andaloro, F. - 1983b. Résume des paramètres biologiques sur Pagellus acarne de la mer Tyrrhénéenne méridionale et de la mer Ionienne septentrionale. FAO Fish. Rep., 266: 89-92.

Andaloro, F. and S. Prestipino Giarritta. - 1985. Contribution to the knowledge of the age, growth and feeding of Pandora, Pagellus erythrinus (L. 1758) in the Sicilian Channel. FAO Fish. Rep., 336: 85-87.

Anonymous. - 2000. Mediterranean Landings Pilot Project - MEDLAND. Final Report. EU project 97/0066.

Bertrand, J.A., L. Gil de Sola, C. Papaconstantinou, G. Relini and A. Souplet. -2000. An international bottom trawl survey in the Mediterranean: the MEDITS programme. In: J.A. Bertrand and G. Relini (eds.), Demersal resources in the Mediterranean, Proceedings of the Symposium held in Pisa, 18-21 March 1998, Actes de Colloques 26, pp. 76-93. IFREMER, Plouzané.

Bertrand, J., L. Gil de Sola, C. Papaconstantinou, G. Relini and A. Souplet. - 2002. The general specifications of the MEDITS surveys. Sci. Mar., 66 (Suppl. 2): 9-17.

Caddy, J.F. - 1997. 5. Mediterranean and Black Sea. Review of the state of world fishery resources: marine fisheries. FAO Fish. Circ., 920: 1-173.

Campillo, A. - 1992. Les pêcheries françaises de Méditerranée: synthèse des connaissances. IFREMER, France.

Cejas, J., S. Jerez, F.J. Santamaría, M. Samper and R. Flores. 1993. Estudios preliminares de la reproducción y cultivo larvario de la breca Pagellus erythrinus en cautividad. In: A. Cerviño, A. Landín, A. Coo, A. Guerra and M. Torre (eds.), Actas IV Congreso Nacional de Acuicultura, pp. 61-65. Villagarcía de Arosa, Spain.

D’Ancona, U. - 1949. Ermafroditismo ed intersessualità nei Teleostei. Experientia, 5: 381-389.

De Gaetani, D. - 1935. Uova, sviluppo embrionale e stadi postembrionali negli sparidi. 3 Pagellus acarne. Mem. Com. Talassogr. Ital., 220: 1-20.

Desbrosses, P. - 1932. La dorade commune (Pagellus centrodontus Delaroche) et sa pêche. Rev. Trav. Inst. Pêches Marit., 5: 167222.

Dremière, P.Y. and L. Fiorentini. - 1996. Mise au point de l'engin de prélèvement. In: Campagne internationale de chalutage démersal en Méditerranée (Medits 95). Vol. I. Rapport général. Bertrand J. Coordonnateur général. Etude 94/047 IFREMER/CE，94/011 IEO/CE，94/057 SIBM/CE， 94/051 NCMR/CE.

Erzini, K., J.M.S. Gonçalves, L. Bentes, P.G. Lino and J. Ribeiro. 1998. Species and size selectivity in a "red" sea bream longline "métier" in the Algarve (southern Portugal). Aquat. Living Resour., 11: 1-11.

Fiorentini, L., J.F. Caddy and J.I. de Leiva. - 1997. Long and shortterm trends of Mediterranean fishery resources. Stud. Rev. GFCM FAO, 69: 1-72.

Girardin, M. and J.P. Quignard. - 1985. Croissance de Pagellus erythrinus (Pisces, Sparidae) dans le Golfe du Lion. Cybium, 9: 359-374.

Greco, S., L. Genovese and V. Micale. - 1995. Growth, gonadal histology and liver lipid composition in Pagellus acarne. Cah. Opt. Médit., 16: 89-102.

Grosslein, M.D. - 1971. Some observation on accuracy of abundance indices derived from research vessel surveys. Redbook ICNAF, 3: 249-266.

Jarboui, O., A. Ghorbel and A. Bouain. - 1998. Stock of common pandora (Pagellus erythrinus) in the Gabes Gulf (Tunisia): fishing situation and management possibilities. Cah. Opt. Médit., 35: 251-260.

Jukic, S. and E. Arneri. - 1984. Distribution of hake (Merluccius merluccius, L.), striped mullet (Mullus barbatus, L.) and pandora (Pagellus erythrinus, L.) in the Adriatic Sea. FAO Fish Rep., 290: 85-91.

Harden Jones, F.R. - 1974. Objectives and problems related to research into fish behaviour. In: F.R. Harden Jones (ed.), Sea Fisheries Research, pp. 261-275. Elek Science, London.

Krug, H.M. - 1990. The Azorean Blackspot Seabream, Pagellus bogaraveo (Brünnich, 1768) (Teleostei, Sparidae): reproductive cycle, hermaphroditism, maturity and fecundity. Cybium, 14: 151-159. 
Larrañeta, M.G. - 1964. Sobre la biología de Pagellus erythrinus L. especialmente de las costas de Castellón. Inv. Pesq., 27: 121146.

Larrañeta, M.G. - 1967. Crecimiento de Pagellus erythrinus de las Costas de Castellón. Inv. Pesq., 31: 185-258.

Lissia Frau, A.L. - 1968. Le manifestazioni della sessualità degli Sparidi. Studi Sassaresi, 4: 243-261

Livadas, R.J. - 1988. A study of the biology and population dynamics of pandora (Pagellus erythrinus L. 1758), family Sparidae in the seas of Cyprus. FAO Fish. Rep., 412: 58-76.

Micale, V. and L. Genovese. - 1998. Supporto della ricerca allo sviluppo di tecniche di acquacoltura per specie innovative: Pagellus erythrinus, Pagellus bogaraveo, Seriola dumerilii. Biol. Mar. Medit., 5(3): 1192-1199.

Mytilinéou, C. - 1988. Données biologiques sur le pageot, Pagellus erythrinus, des côtes orientales de la Grece centrale. FAO Rapp. Pêch, 412: 77-82.

Orsi Relini, L. and B. Fida. - 1992. Note di biologia di Pagellus bogaraveo in Mar Ligure. Oebalia, 17 (suppl.): 81-86.

Orsi Relini, L., G. Relini, G. Romeo and L. Tunesi. - 1984. Reclutamento e primo accrescimento degli Sparidi del genere Pagellus sui fondi strascicabili costieri del Mar Ligure. Nova Thalassia, 6 (suppl.): 489-501.

Orsi Relini, L. and G. Romeo. - 1985. Vertical distribution, age, growth and mortality of Pagellus erythrinus on trawled areas of the Ligurian Sea. Rapp. Comm. int. Mer Médit., 29 (8): 103106.

Pajuelo, J.G. and J.M. Lorenzo. - 1998. Population biology of the common pandora Pagellus erythrinus (Pisces: Sparidae) off the Canary Islands. Fish. Res., 36: 75-86.

Pauly, D. - 1983. Algunos métodos simples para la evaluación de recursos pesqueros tropicales. FAO Doc. Tec. Pesca, 234: 1-49.

Pennington, M. and T. Strømme. - 1998. Surveys as a research tool for managing dynamic stocks. Fish. Res., 37: 97-106.

Petrakis, G. and K.I. Stergiou. - 1996. Gill net selectivity for four fish species (Mullus barbatus, Pagellus erythrinus, Pagellus acarne and Spicara flexuosa) in Greek waters. Fish. Res., 27 (13): $17-27$.

Rijavec, L., and S. Zupanovic. - 1965. A contribution to the knowledge of the biology of P. erythinus L, in the Middle Adriatic. Rapp. P.-V Réun. CIESM, 18(2): 195-200.

Sánchez, F. - 1983. Biology and fishery of the red seabream (Pagellus bogaraveo, B.) in VI, VII and VIII subareas of ICES ICES C.M. G/38: pp. 11

Santos, M.N., C.C. Monteiro and K. Erzini. - 1995. Aspects of the biology and gillnet selectivity of the axillary seabream (Pagellus acarne, Risso) and common pandora (Pagellus erythrinus, Linnaeus) from the Algarve (south Portugal). Fish. Res., 23: 223-236.

Souplet, A. - 1996. Définition des estimateurs. In: Campagne internationale de chalutage démersal en Méditerranée (Medits 95). Vol. III. Indices de biomasse et distributions en tailles. Bertrand J. Coordonnateur général. Etude 94/047 IFREMER/CE, 94/011 IEO/CE, 94/057 SIBM/CE, 94/051 NCMR/CE.

Sousa, F., E. Isidro and K. Erzini. - 1999. Semi-pelagic longline selectivity for two demersal species from the Azores: the black spot sea bream (Pagellus bogaraveo) and the bluemouth rockfish (Helicolenus dactylopterus dactylopterus). Fish. Res., 41: 25-35.

Spedicato, M.T., M. Contegiacomo, P. Carbonara and G. Lembo. 1998. Sistemi di produzione innovativi orientati alla maricoltura di nuove specie: Pagellus erythrinus, Pagellus bogaraveo. Biol. Mar. Medit., 5(3): 1180-1185.

Stergiou, K.I., E.D. Christou, D. Georgopoulous, A. Zenetos and C. Souvermezoglou. - 1997. The Hellenic seas: physics, chemistry, biology and fisheries. Oceanogr. mar. Biol. ann. Rev., 35: 415-438.

Tortonese, E. - 1975. Osteichthyes, Pesci ossei, Fauna d'Italia, Vol. XI., Ed. Calderini, Bologna.

Vassilopoulou, V.., C. Mytilinéou and C. Papaconstantinou. - 1986. Preliminary biological data on the red pandora (Pagellus erythrinus) in the Greek seas. FAO Fish. Rep., 361: 107-112. 